\title{
LA DIMENSIONE "POLITICA" DELLA GIURISPRUDENZA DELLA CORTE COSTITUZIONALE IN ITALIA
}

\author{
The "political" dimension of the jurisprudence \\ of the Constitutional Court in Italy \\ GIANCARLO ROLLA \\ Università degli Studi di Genova \\ rolla@unige.it
}

Cómo citar/Citation

Rolla, G. (2019)

La dimensione "politica" della giurisprudenza della Corte Costituzionale in Italia. Anuario Iberoamericano de Justicia Constitucional, 23(2), 299-338. doi: https://doi.org/10.18042/cepc/aijc.23.09

\section{Resumen}

El artículo analiza el papel desempeñado por la Corte Costituzionale italiana desde su configuración constitucional en los inicios de la república y su progresiva evolución, tanto en lo relativo a sus relaciones con el Parlamento o las regiones, como en relación con los derechos sociales y sus vínculos económico-financieros.

\section{Palabras clave}

Corte constitucional; política; jurisprudencia; democracia.

\section{Abstract}

The article analyses the role played by the Italian Constitutional Court since its constitutional configuration at the beginning of the republic and its progressive evolution, both in relation to its relations with Parliament or the Regions, and in relation to the social rights and their economic-financial ties.

\section{Keywords}

Constitutional Court; politics; jurisprudence; democracy. 
I. LA PROGRESSIVA MESSA A FUOCO DELLA NATURA DELLA CORTE COSTITUZIONALE. II. LA FASE DI AVVIO DELL'ESPERIENZA ITALIANA DI GIUSTIZIA COSTITUZIONALE. III. LA FASE DELLA "MEDIAZIONE" E IL POTENZIAMENTO DELLE TECNICHE INTERPRETATIVE E DI DECISIONE. IV. DUE CASI DI CONTROVERSO RAPPORTO TRA IL GIUDICE COSTITUZIONALE E IL PARLAMENTO. V. IL DIFFICILE EQUILIBRIO TRA GARANZIA DEI DIRITTI SOCIALI E VINCOLI ECONOMICO-FINANZIARI. VI. LA CORTE COSTITUZIONALE COME GARANTE DELL' UNITÀ DELL'ORDINAMENTO E ARBITRA NEI RAPPORTI TRA LO STATO E LE REGIONI. VII. LA GIUSTIZIA COSTITUZIONALE DINANZI ALLE ASPIRAZIONI IDENTITARIE DI ALCUNI TERRITORI. VIII. LA CORTE COSTITUZIONALE INNANZI AI LIMITI DELLA DEMOCRAZIA RAPPRESENTATIVA. BIBLIOGRAFIA.

\section{LA PROGRESSIVA MESSA A FUOCO DELLA NATURA DELLA CORTE COSTITUZIONALE}

I costituenti hanno introdotto nella Seconda parte della Costituzione tre fondamentali novità finalizzate ad affermare il principio organizzativo della separazione dei poteri: il Consiglio superiore della magistratura (che sottrae al Ministro della giustizia importanti poteri al fine di garantire l'autonomia e l'indipendenza dell'ordine giudiziario), le Regioni (che danno vita a un cheek and balance di tipo verticale) e la Corte costituzionale, la cui prima sentenza risale al 1956. La dottrina, sin dai primi commenti alla Costituzione, comprese la rilevanza e le potenzialità di questo organo, che poteva favorire la legalità sostanziale del sistema normativo (sia depurandolo dalle possibili antinomie, sia assolvendo a una funzione di interpretazione autentica del significato delle disposizioni costituzionali), assicurare la tutela dei diritti fondamentali, garantire l'equilibrio istituzionale tra i poteri dello Stato e tra questo e gli enti politici decentrati (Ambrosini, 1953; Cappeletti, 1955; Battaglini, 1957; Baschieri et al., 1949; Calamandrei y Levi, 1950; D’Orazio, 1981; Bonini, 1996; De Siervo, 2008).Se è indubbio che questa istituzione seppe acquisire una salda legittimazione sociale e godere di una piena autorevolezza all'interno della forma di governo italiana, non va tuttavia dimenticato che ciò fu il risultato soprattutto della qualità complessiva della sua giurisprudenza, della 
capacità di assecondare nel tempo l'evoluzione della società e di dipanare con equilibrio i principali nodi istituzionali. Tale esito non era affatto era scontato, dal momento che dal dibattito in Assemblea costituente non emerse una visione omogenea e condivisa del ruolo, delle attribuzioni e dei criteri di composizione della Corte costituzionale: il disegno originario si presentava, come autorevolmente affermato, con i "connotati dell'incertezza e dell'ambiguità" (Modugno, 1985: 534).

La soluzione che emerse dal testo della Costituzione fu condizionata più che dal peso di posizioni di principio contrarie a prevedere forme di controllo delle leggi da parte dei giudici - da obiezioni che ponevano in guardia circa l'opportunità di attribuire a una organo di natura giurisdizionale, non rappresentativo la competenza di instaurare - come ebbe a dire Francesco Carnelutti- una sorta di "processo al legislatore", depotenziando la centralità del Parlamento, espressione diretta ai sensi dell'art. 1 Cost. della sovranità popolare (Carnelutti, 1959: 1; D’Orazio, 1981). Né va trascurato che la maggioranza favorevole all'istituzione della Corte costituzionale se, per un verso, coagulò un consenso attorno ad alcuni criteri di carattere generale (la rigidità costituzionale, l'introduzione di un procedimento ad hoc finalizzato verificare il rispetto delle norme costituzionali, l'affidamento di tale compito a un apposito organo di rilievo costituzionale), per un altro verso si differenziò al suo interno circa il modo organizzare siffatto tipo di controllo (Pizzorusso, 1981: 64).

Una prima posizione - sostenuta in particolare da Calamandrei- proponeva un ibrido tra l'esperienza nordamericana di judicial review e il controllo astratto di derivazione austriaca: la funzione di giudice costituzionale avrebbe dovuto essere ripartita tra i giudici comuni (che risolvevano il contrasto tra norme ordinarie e Costituzione nel corso di un processo, con effetti inter partes) e la Corte costituzionale (che decideva sulla base di un ricorso presentato, entro tre anni dall'entrata in vigore della legge, da un Procuratore generale su richiesta di parlamentari o dei cittadini al determinarsi di particolare presupposti). Le decisioni del giudice costituzionale avrebbero vincolato il Parlamento a modificare gli eventuali vizi di incostituzionalità.

Un'altra - caldeggiata in particolare da Leone- prevedeva un ricorso accentrato e successivo nei confronti non solo delle leggi, ma anche dei regolamenti e degli atti amministrativi, che poteva essere presentato da un ampio ventaglio di organi (Presidente della Repubblica, Governo, Presidente della Regione e del Consiglio regionale, un organo del potere giudiziario, cittadini che ne abbiano interesse); inoltre, la dichiarazione di incostituzionalità aveva effetti retroattivi, salva la possibilità per la Corte costituzionale di dettare norme transitorie valide nei sei mesi successivi alla pubblicazione della sentenza. 
A sua volta il costituente Einaudi ipotizzò di distinguere il controllo di legittimità delle norme dai conflitti di competenza: il primo avrebbe dovuto essere di competenza dei giudici comuni, i secondi erano riservati alla Corte di cassazione. Invece, le competenze di natura prettamente politica (come il giudizio sui reati del Presidente della Repubblica e dei ministri) sarebbero spettate al Parlamento in seduta comune. Infine, Mortati suggerì di valorizzare il ruolo della Corte costituzionale come "giudice dei diritti", ipotizzando una sorta di recurso de amparo constitucional).

$\mathrm{Su}$ questi presupposti, fu assai difficile conseguire, al termine dei lavori costituenti, una sintesi tra le diverse, eterogenee proposte, che riuscirono a coagularsi soltanto attorno all'idea che la Corte costituzionale dovesse qualificarsi come organo di garanzia della Costituzione, non antagonistico rispetto alla centralità del Parlamento, ma in grado di affiancarlo fornendo un'interpretazione delle leggi secundum Constitutionem (Fioravanti, 1998: 107; Repetto, 2018: 730).

Inoltre, anche se risultò evidente l'influenza del prototipo introdotto dalla Costituzione austriaca del 1920 (Calamandrei, 1950; Rolla, 2017: 839), il sistema effettivo di giustizia costituzionale non si limitò a una mera applicazione di un modello storico, ma tenne conto di diversi elementi.

Innanzitutto della specificità dell'interpretazione delle disposizioni delle Costituzioni democratiche e sociali, dal momento che in esse sono numerose le norme a contenuto giuridico indeterminato (il cui contenuto normativo richiede la sussunzione di elementi propri di discipline non giuridiche), le disposizioni polisenso (la cui interpretazione è aperta all'evoluzione normativa), le disposizioni programmatiche (che orientano la successiva attività normativa dei pubblici poteri), le disposizioni "pattizie" (frutto della mediazione tra tendenze culturali diverse, codificate come patto tra le componenti principali di una società pluralistica), nonché quelle tese a favorire l'elasticità dell'ordinamento costituzione consentendogli di adattarsi al variare della società).

Di conseguenza, maturò la consapevolezza che l'interpretazione costituzionale si dovesse differenziare da quella norme legislative e che questo compito potesse essere assolto solo da un giudice "particolare", sensibile all'evoluzione della vita sociale, politica ed economica del paese, attento agli effetti che le sue decisioni possono produrre nel contesto sociale e istituzionale. In altri termini, la Corte costituzionale doveva qualificarsi come un giudice sui generis: un organo indipendente dalla politica, ma non estraneo al circuito politico e istituzionale, in grado di attuare ma anche implementare la Costituzione, facendo di essa un living tree.

Nel medesimo tempo - e in apparente contraddizione con quanto detto sopra- la legislazione attuativa del titolo VI della Costituzione ha favorito, 
grazie all'introduzione della "questione incidentale di costituzionalità", l'instaurarsi di un continuum tra l'attività interpretativa del giudice costituzionale e quella dei giudici comuni ${ }^{1}$, che ha contribuito ad allontanare progressivamente il sistema italiano di giustizia costituzionale dal suo prototipo di riferimento dando vita a un sistema essenzialmente ibrido: infatti la giurisdizione costituzionale attivata da una "questione" sollevata da un giudice nel corso di un processo fa sì che il giudizio costituzionale, pur essendo per natura oggettivo - finalizzato, cioè, a valutare la coerenza di una norma con le disposizioni costituzionali- non può trascurare che gli esiti dello stesso debbono essere "rilevanti" sia per il processo che ha occasionato la questione di costituzionalità, sia per altri procedimenti giurisdizionali (in corso o futuri) (Pegoraro, 1998: 27; Aragón, 1979: 174).

Di conseguenza, a mano a mano che il giudizio incidentale di costituzionalità ha incrementato la sua rilevanza sotto il profilo numerico e della varietà delle problematiche affrontate, il giudizio di costituzionalità ha dovuto affinare i meccanismi procedurali per evitare l'insorgere di conflitti interpretativi tra le diverse giurisdizioni. A tale risultato si è pervenuti progressivamente sulla base di successive approssimazioni, attraverso un "dialogo" continuo tra le giurisdizioni (Rolla, 2010: 1865; Caretti y Gardino, 1988; De Siervo, 2005; Mannella, 2011). Prima, si è distinto tra l'interpretazione della normativa costituzionale (riservata al giudice costituzionale e vincolante per tutti gli operatori del diritto) e il significato della legislazione ordinaria, che deve emergere dal c.d. "diritto vivente", cioè da una "consolidata interpretazione giurisprudenziale, suffragata dall'esistenza di almeno una pronuncia della Corte di cassazione" ${ }^{2}$. Quindi si è precisato che spetta al giudice comune, nell'esercizio dei suoi poteri interpretativi, pervenire a un'interpretazione delle disposizioni secundum Costitutionem, mentre si deve coinvolgere il giudice costituzionale soltanto se è impossibile realizzare un'interpretazione costituzionalmente corretta $^{3}$.

In definitiva, si può ritenere che il giudice costituzionale abbia assunto nel tempo le sembianze di un "Giano" i cui due volti gli consentono di guardare sia il passato (il testo della Costituzione) che il futuro (la sua evoluzione e trasformazione nel tempo), sia il contesto politico che i rapporti con la giurisdizione comune.

Soprattutto la legge costituzionale n. 1 del 1948 e la legge ordinaria n. 87 del 1953.

Ad esempio, nella sentenza n. 356 del 1996

Ad esempio, nella ordinanza n. 19 del 2003. 


\section{LA FASE DI AVVIO DELL'ESPERIENZA ITALIANA DI GIUSTIZIA COSTITUZIONALE}

La fase di avvio dell'esperienza di giustizia costituzionale ebbe una duplice, importante caratterizzazione: l'una di natura processuale inerente alle tecniche processuali, l'altra di natura sostanziale relativa al contenuto delle sue prime decisioni.

La prima è riconducibile alla circostanza che la Corte costituzionale italiana, specie se confrontata con altre giurisdizioni costituzionali, può esercitare un numero limitato di competenze, in conseguenza di una precisa scelta dei costituenti che vollero mettere la sua azione al riparo da un'eccessiva esposizione "politica" riducendo le possibilità di "conflitto" con le istituzioni rappresentative. Inoltre, nei primi venti anni di funzionamento, il canale di accesso alla Corte fu rappresentato dall'ordine giudiziario: si consideri che il contenzioso tra lo Stato e le Regioni decollò solo in seguito all'attivazione delle Regioni ordinarie nel 1971, che la prima decisione in materia di conflitti tra poteri dello Stato si ebbe con la sentenza n. 13 del 1975, così come a tale periodo risalgono anche le prime decisioni in tema di ammissibilità del referendum abrogativo.

Di conseguenza, nei primi anni di attività le "chiavi" per aprire la "porta" di accesso al giudizio costituzionale sono state nelle mani dei giudici ordinari, dalla cui discrezionalità dipendeva la scelta se sollevare nel corso di un processo una questione di legittimità costituzionale, con il rischio che tale fondamentale organo di garanzia della legalità costituzionale finisse per svolgere un ruolo marginale nella dinamica istituzionale della Repubblica.

Va ascritto, quindi, a merito della Corte costituzionale il risultato di aver individuato particolari tecniche decisorie, soprattutto di natura processuale con l'obiettivo di favorire un ampio accesso al giudizio di legittimità costituzionale (Luciani, 1984). Esse si sono orientate essenzialmente in tre direzioni.

Innanzitutto, la Corte ha reso giustiziabili tutte le disposizioni della Costituzione, segnando un punto di rottura rispetto a qualificati orientamenti della dottrina e della giurisprudenza — specie della Corte di Cassazione e del Consiglio di Stato- i quali avevano graduato l'efficacia delle disposizioni costituzionali, introducendo una suddivisione di massima tra norme immediatamente applicabili, norme non suscettibili di immediata applicazione in quanto rinviano al legislatore una più concreta determinazione, disposizioni generali che contengono mere direttive nei confronti del legislatore ${ }^{4}$. In tal

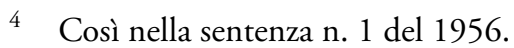


modo, ha definitivamente depotenziato ai fini del giudizio di legittimità costituzionale l'utilità della distinzione tra norme precettive e programmatiches.

Inoltre, ha ulteriormente ampliato il parametro del proprio giudizio estendendolo ai principi che "appartengono all'essenza dei valori supremi sui quali si fonda la Costituzione italiana": cioè a norme non scritte, ricavabili da un'analisi sistematica del testo costituzionale, che si pongono "al di sopra" delle singole disposizioni e rappresentano per esse un limite sostanziale sindacabile dal giudice costituzionale ${ }^{6}$.

In secondo luogo, il giudice costituzionale ha riconosciuto la propria competenza a "giudicare sulle controversie relative alla legittimità costituzionale delle leggi e degli atti aventi forza di legge anche se anteriori alla entrata in vigore della Costituzione". In tal modo il controllo di costituzionalità è stato esteso a tutte le norme di rango legislativo vigenti, consentendo di annullare molte disposizioni emanate durante il periodo della dittatura e contrarie alla nuova Costituzione (Mortati, 1972: 821; Rolla, 2018: 469).

Siffatto orientamento ha differenziato l'ordinamento italiano da altri ordinamenti europei: si consideri che nella Repubblica federale di Germania le leggi anteriori alla prima riunione del Bundestag restavano in vigore e la valutazione della compatibilità con la Legge fondamentale spettava al giudice comune; mentre, secondo il terzo comma della Disposizione Derogatoria della Costituzione spagnola il contrasto tra Costituzione e legge anteriore può essere risolto dal giudice comune in sede di giudizio sull'applicabilità di questa ultima. In entrambi i casi, quindi, le decisioni avevano soltanto effetti inter partes e non erga omnes come nel caso delle sentenze della Corte italiana.

In terzo luogo, il giudice costituzionale si è premurato di evitare che il meccanismo principale di accesso al giudice costituzionale (la questione di costituzionalità) si trasformasse in un indebito ostacolo al pieno dispiegarsi del controllo di costituzionalità. A tal fine, ha fornito un'interpretazione estensiva della nozione di giudice a quo: ad esempio, nella sentenza n. 84 del 1966, ha precisato che l'espressione "giudizio" è comprensiva di tutti i vari procedimenti di carattere decisorio svolti dal titolare di un ufficio giurisdizionale, mentre possono rientrare nella nozione di "autorità giurisdizionale" anche gli organi

5 In precedenza, la dottrina prevalente - anche alla luce di un'importante sentenza delle Sezioni unite penali della Corte di Cassazione, che nel febbraio del 1948 distingueva, nell'ambito delle disposizioni costituzionali, tra norme precettive e norme programmatiche - riteneva che per dare forza normativa a molte statuizioni della Costituzione, necessaria l'interpositio legislatoris (García de Enterría, 1985: 68; Crisafulli, 1993).

6 Si veda, ad esempio, la sentenza n. 1146 del 1988 (Casavola, 1955: 1555; Modugno, 2000: 95; Razzano, 2002; Ventura y Morelli, 2015). 
estranei all'ordine giudiziario che siano investiti di funzioni giudicanti e si trovino in posizione super partes ${ }^{7}$. In tal modo ha ampliato la partecipazione alla giurisdizione costituzionale - estendendola oltre i confini della giurisdizione comune-; mentre si è rivelata assai più cauta nel legittimare l'intervento nel procedimento a soggetti privi dello status di parte, limitandola a "terzi titolari di un interesse qualificato, inerente in modo diretto e immediato al rapporto sostanziale dedotto in giudizio" 8 .

Il contributo innovativo apportato nell'iniziale esperienza di giustizia costituzionale attiene soprattutto alla qualità delle sue prime decisioni. Infatti, nel periodo che va dal 1956 alla metà degli anni '70, il giudice costituzionale ha sopperito ai ritardi del Parlamento nell'abrogare la legislazione anteriore alla nascita della Repubblica, assumendo un ruolo di primo piano nella democratizzazione dell'ordinamento giuridico italiano e annullando molte disposizioni illiberali approvate nel corso della Monarchia e del Fascismo. La sua azione si è rivelata, in questa fase, coerente con le intenzioni originarie dei Costituenti, favorevoli ad affidare a questo organo il compito di allineare il sistema legale alle nuove disposizioni costituzionali, evitando conflitti con il legislatore repubblicano.

Rilevante è stato, ad esempio, l'impatto (anche sociale) delle sue sentenze a tutela della libertà di manifestazione del pensiero, che viene depurata delle più odiose eredità del fascismo, quali le molte autorizzazioni di polizia (sentenza n. 1 del 1956); della eguaglianza tra i sessi, dichiarando incostituzionale una disposizione del 1919 che escludeva le donne da una vasta categoria di impieghi pubblici (sentenza n. 33 del 1960); della libertà di riunione, per cui non vi è più l'obbligo di preavviso per le riunioni in luogo aperto al pubblico (sentenza n. 90 del 1970); della libertà di comunicazione, nel senso che le intercettazioni telefoniche debbono avvenire sempre sotto il controllo del giudice, che le deve adeguatamente motivare (sentenza n. 100 del 1968 e n. 34 del 1973).

Così come è stata apprezzata la sua giurisprudenza in tema di diritto di sciopero, in cui ha precisato che le finalità di tale istituto non riguardano soltanto il rapporto di lavoro, ma si estendono anche a qualsiasi interesse dei lavoratori (sentenze n. 123 del 1962 e n. 141 del 1967); ovvero quella relativa al diritto alla salute, riconoscendo che la gravidanza può essere interrotta qualora la gestazione implichi un danno o pericolo grave per la salute della madre (sentenza n. 27 del 1975); o alla legittimità della carcerazione preventiva, i cui

Sulla nozione di giudice a quo: Patroni, 2012; Oddi, 2007: 28; Pinelli, 2000: 618.

8 Vedi sentenza n. 194 del 2018 (Groppi, 2019). 
limiti massimi debbono essere tali da non vanificare il principio di presunzione di non colpevolezza (sentenza n. 64 del 1970).

Grazie alla sua giurisprudenza in tema di diritti, il giudice costituzionale ha esercitato un'importante funzione di educazione civica - contribuendo a permeare la società dei valori costituzionali- e fu immediatamente percepito come il principale difensore della Costituzione, dal momento che il Parlamento nelle prime legislature si mostrò molto timido nell'abrogare la legislazione prerepubblicana. In tale azione risiedono i motivi dell'autorevolezza acquisita da quest'organo all'interno della forma di governo italiana.

E' impossibile non riconoscere il suo ruolo fondamentale nel disboscare dall'ordinamento italiano la legislazione autoritaria risalente al periodo fascista, anche se non deve trascurare che su alcuni temi sensibili la Corte costituzionale ha evidenziato una certa difficolta ad entrare in sintonia con un nuova sensibilità che iniziava a penetrare all'interno della società italiana. Ad esempio, non colse con l'auspicata celerità alcune modificazioni del costume relative ai rapporti tra $\mathrm{i}$ coniugi all'interno della famiglia o inerenti alla sfera sessuale degli individui.

Rispetto alla normativa penale che puniva l'adulterio della sola moglie la Corte, in un primo momento, aveva giustificato tale differenziazione di trattamento ritenendo il principio costituzionale dell'unità familiare preminente rispetto all'uguaglianza giuridica e morale dei coniugi sulla base di un pregiudizio sociale per cui l'infedeltà della moglie era considerata socialmente più grave di quella del marito? . Mentre, in un primo momento, ha giustificato il divieto di propagandare le pratiche anticoncezionali qualora $\mathrm{i}$ modi di manifestazione del pensiero risultassero in contrasto con la morale ${ }^{10}$. Solo successivamente, in un differente contesto culturale, la Corte ha mutato orientamento, ritenendo il principio di eguaglianza tra i coniugi prevalente rispetto a quello di unità familiare (sentenza n. 126 del 1968) e dichiarando la non contrarietà alla Costituzione della propaganda dei metodi contracettivi (sentenza n. 49 del 1971).

\section{LA FASE DELLA "MEDIAZIONE" E IL POTENZIAMENTO DELLE TECNICHE INTERPRETATIVE E DI DECISIONE}

Nel periodo successivo alla seconda metà degli anni ' 70 si modifica profondamente il contesto istituzionale in cui il giudice costituzionale opera, im-

\footnotetext{
$9 \quad$ Si veda la sentenza n. 64 del 1961.

10 Si veda la sentenza n. 9 del 1965.
} 
ponendo alla Corte una continua rilegittimazione nei confronti della società: come è stato autorevolmente sottolineato, pur avendo prodotto un grande acquis giurisprudenziale, non poteva "vivere di rendita" (Elia, 2009: 130).Diversi fattori hanno contribuito a definire i caratteri di questa nuova fase.

Innanzitutto, il giudice costituzionale ha potuto esercitare appieno l'intero ventaglio delle attribuzioni attribuitegli dalla Costituzione (con l'eccezione della messa in stato d'accusa del Presidente della Repubblica). Le sue decisioni non si limitano alle sole questioni di costituzionalità, ma si estendono alla risoluzione di un ampio contenzioso tra lo Stato e le Regioni, ai conflitti tra i poteri dello Stato e ai giudizi di ammissibilità delle richieste di referendum abrogativo. Queste competenze incrinano il precedente monopolio del rapporto Corte - giudici comuni e impongono al giudice costituzionale di affiancare, a un rapporto collaborativo con i giudici comuni ${ }^{11}$. una difficile dialettica con il legislatore, con gli altri poteri dello Stato, con le Regioni, con il corpo elettorale e i promotori dei referendum, con le giurisdizioni sovranazionali.

In secondo luogo, l'oggetto delle sue decisioni non si limitava alla legislazione anteriore alla Costituzione, ma comprendeva con (frequenza sempre maggiore) leggi da poco approvate dal Parlamento repubblicano o dai Consigli regionali: di conseguenza, non si trattava più di depurare il diritto vigente dalle norme contrarie alla Costituzione, bensì di valutare la conformità a Costituzione di scelte politiche prese dalle assemblee rappresentative. In diversi casi il confine tra discrezionalità e illegittimità si è rivelato incerto o assai poroso; inoltre, non va dimenticato che le moderne società pluralistiche sono percorse da interessi non omogenei, da tensioni non sempre componibili, per cui la decisione politica deve con frequenza maggiore bilanciare le situazioni soggettive suscettibili di entrare in conflitto oppure contemperare l'esercizio di un diritto con la salvaguardia di un principio o di un valore costituzionale. Inoltre, non sempre l'equilibrio tra aspettative contrastanti perseguito dal legislatore (definitional balancing) trova conferma nella valutazione del giudice allorché decide sulle singole fattispecie (ad hoc balancing).

In questi casi il contenzioso costituzionale si conclude con la ricerca di una mediazione equilibrata tra i diversi interessi sottesi alla questione di costituzionalità e la Corte deve valutare le scelte del legislatore con riferimento ai principi di ragionevolezza o di proporzionalità (Bin, 1992). Nel primo caso ritiene incostituzionali le scelte legislative che regolano situazioni differenti secondo criteri arbitrari, determinando un' irrazionale contraddizione tra la

11 Vedi supra, paragrafo 1. 
finalità della legge e il contenuto delle norme ${ }^{12}$; nel secondo caso, invece, evita che il godimento di un diritto avvenga con modalità tali da comprimere i diritti altrui "oltre misura", cioè oltre quanto è necessario per esercitare un proprio diritto (Bernal, 2003; Heintzen, 2012; Barak, 2012; Morrone, 2014).

Ad esempio, in materia di diritto alla salute, il giudice costituzionale ha precisato che il diritto a ottenere determinati trattamenti sanitari è condizionato dall'attuazione che il legislatore ordinario ne dà attraverso il bilanciamento dell'interesse tutelato con gli "altri interessi costituzionalmente protetti, tenuto conto dei limiti oggettivi che lo stesso legislatore incontra nella sua opera di attuazione in relazione alle risorse organizzative e finanziarie di cui dispone al momento"13.

L'esigenza di valutare le norme non solo sotto il profilo della mera legittimità, ma anche con riferimento alla ragionevolezza della scelta legislativa e alla sua capacità di contemperare in modo equilibrato interessi e diritti contrastanti ha imposto al giudice costituzionale di graduare il proprio atteggiamento a seconda delle situazioni concrete e di fissare, di volta in volta, la linea di demarcazione tra discrezionalità politica e illegittimità costituzionale. Nello svolgimento di questo compito la Corte, non trovando un adeguato supporto nella legislazione che regola il processo costituzionale, ha dovuto sopperire alla limitatezza degli strumenti decisionali messi a disposizione dal legislatore con una giurisprudenza "creativa" che ha dato vita a una varietà di tipi di sentenze di natura processuale.

Ad esempio, al fine di mettere un certo lasso di tempo a disposizione del Parlamento (per approvare una nuova disciplina della materia) o delle pubbliche amministrazioni (per meglio fronteggiare organizzativamente gli effetti di una decisione di illegittimità costituzionale) ha dato vita alle pronunce di "incostituzionalità differita": in questi casi, il giudice costituzionale, bilanciando diverse esigenze di rilievo costituzionale, individua il dies a quo degli effetti di annullamento ${ }^{14}$. Speculari a questo tipo di decisioni sono le decisioni di illegittimità sopravvenuta con le quali il vizio della legge non decorre ab origine, ma partire da un momento successivo determinato dal giudice costituzionale ${ }^{15}$.

Sono frequenti anche le situazioni in cui la Corte, per assicurare la tutela effettiva di diritti sociali o per evitare discriminazioni contrarie al principio

12 Sul principio di ragionevolezza, Moscarini, 1996; Paladin, 1997: 899; Scaccia, 2000; Modugno, 2007; Morrone, 2000; D’Andrea, 2005.

13 Ad esempio la sentenza n. 455 del 1990.

14 Vedi la sentenza n. 119 del 1981.

15 Ad esempio, la sentenza n. 50 del 1989. In dottrina, Ruotolo, 2010; Pinardi, 1993; D’Amico, 1993; Politi, 1997. 
di eguaglianza, decide di sostituirsi al legislatore ricorrendo alle sentenze c.d. "additive", con le quali la dichiarazione di incostituzionalità colpisce una disposizione "nella parte in cui non prevede" o "non estende ad altri soggetti una determinata prestazione" 16 .

In altri casi, il giudice costituzionale ha preferito ricercare (ove possibile) un "dialogo" con il Parlamento, tendendo presente che il controllo di legittimità della Corte costituzionale deve escludere "ogni valutazione di natura politica e ogni sindacato sull'uso del potere discrezionale del Parlamento" ${ }^{17}$. In particolare, si è premurato di evitare che la giurisprudenza costituzionale possa innescare un circuito decisionale parallelo (se non alternativo) a quello della rappresentanza politica. ed ha individuato criteri argomentativi idonei a conseguire un accettabile equilibrio tra sindacato di costituzionalità e supremacy of Parliament (Romboli, 2017).

Tuttavia, se l'obiettivo perseguito dal giudice costituzionale appare chiaro, ciononostante il suo atteggiamento nei confronti del legislatore non sempre è univoco, risultando influenzato sia da fattori "interni" al processo (come l'oggetto della questione di costituzionalità, l'esistenza di precedenti o la consapevolezza dell'opportunità di introdurre un revirement giurisprudenziale), che da altri "esterni" (ad esempio, il contesto politico-istituzionale in cui ha luogo la decisione, la volontà del Parlamento di dare un legislative sequel agli "indirizzi" presenti nelle rationes decidendi della Corte costituzionale, l' attitudine di quest'ultima a esercitare un accorto self-restraint nel valutare situazioni in cui il margine di distinzione tra legittimità e discrezionalità politica risulta assai stretto).

Le posizioni assunte dalla giurisprudenza costituzionale nei confronti del legislatore sono graduabili secondo una scala che va da un elevato grado di deferenza a un atteggiamento propositivo, in cui la Corte si sostituisce, in virtù di specifiche tecniche decisionali, alla latitanza che il Parlamento dimostra dinanzi alla necessità costituzionale di disciplinare una determinata materia.

In diverse situazioni, il giudice costituzionale ha sostanzialmente allineato la sua giurisprudenza alle scelte del legislatore nazionale: ciò è avvenuto, ad esempio, dinanzi ai processi di centralizzazione indotti dalle crisi economiche globali, che la Corte ha assecondato soprattutto quando essi si richiamano (più o meno esplicitamente) ai vincoli conseguenti all'adesione all'Unione eu-

16 Ciò è avvenuto con frequenza in materia di pubblico impiego, di diritto del lavoro e di previdenza sociale, di assistenza e di sanità pubblica.

17 Così l'art.28 della legge n. 87 del 1953. Sui rapporti con il legislatore, Passaglia, 2011; Aja, 1998. 
ropea ${ }^{18}$; oppure quando ha dovuto affrontare questioni controverse — dotate di un elevato tasso di politicità- in cui ha preferito che il vizio di costituzionalità fosse eliminato direttamente dal Parlamento, evitando che un probabile annullamento possa determinare un vuoto normativo, una lacuna giuridica.

Le espressioni linguistiche utilizzate in proposito sono assai varie (dal mero auspicio di revisione legislativa alla dichiarazione di incostituzionalità accertata ma non dichiarata o di "costituzionalità provvisoria"), ma possono essere riassuntivamente ricondotte alla formula delle c.d. sentenze "monito", con le quali il giudice costituzionale introduce una scissione logica tra il dispositivo e la motivazione: il primo determina il rigetto della questione di costituzionalità, la seconda - invece- lascia chiaramente intendere che la normativa impugnata presenta fondati dubbi di compatibilità con il dettato costituziona$\mathrm{le}^{19}$. Nella sostanza, il giudice costituzionale per non creare "vuoti" nel sistema normativo rivolge "un monito" al Parlamento affinché sani con l'approvazione di una nuova normativa il vizio di incostituzionalità, preannunciando in caso di inerzia una futura sentenza di incostituzionalità (Pegoraro, 1987; Pinardi, 1993: 73; D’Orazio, 1967: 134; Modugno, 1981: 1646; Ruggeri, 1988).

In altre situazioni interviene con modalità più sofisticate attraverso"sentenze additive di principio", con le quali il giudice costituzionale precisa i termini della collaborazione con il legislatore: l'uno indica il principio a cui rifarsi per evitare un vizio di incostituzionalità, l'altro deve individuare, nella sua discrezionalità politica, i modi con cui dare attuazione agli indirizzi della Corte e trovare le relative coperture economiche ${ }^{20}$.

18 A titolo di esempio, possiamo richiamare la sentenza n. 198 del 2012 in cui il giudice costituzionale ha confermato la propria giurisprudenza secondo cui il legislatore statale può validamente imporre alle Regioni limiti generali di spesa e fissare criteri di bilancio orientati a ridurre le perdite finanziarie anche nelle materie rientranti nella loro competenza legislativa riservata. Mentre nella sentenza n. 198 del 2008 ha giustificato la capacità delle leggi statali di limitare l'autonomia statutaria delle Regioni al fine di porre un freno alle spese degli organi politici delle Regioni: nel caso di specie, il legislatore avere fissato un limite massimo agli emolumenti dei consiglieri regionali e al numero dei consiglieri e degli assessori.

19 Una vasta eco ha suscitato la sentenza in cui il giudice costituzionale, in materia di sistema radiotelevisivo, ha dichiarato illegittimo non tanto il monopolio in sé, quanto le modalità con le quali era regolato e esercitato, provvedendo — altresì- ad evidenziare alcuni requisiti che avrebbe dovuto possedere una nuova disciplina della materia (sentenza n. 225 del 1974). A tali criteri il Parlamento si è prontamente adeguato approvando la legge di riforma del sistema n. 103 del 1975.

20 Vedi, ad esempio, la sentenza n. 243 del 1993. 
Questo atteggiamento di self- restraint, tuttavia, viene meno dinanzi alle "colonne d'Ercole" rappresentate dalla necessità di salvaguardare alcuni diritti nei casi in cui il mancato intervento del legislatore rende concretamente non fruibile un determinato diritto costituzionalmente tutelato.

In altri termini, il giudice costituzionale deve sopperire alle omissioni del legislatore, sanzionando il mancato rispetto da parte del Parlamento del "dovere di protezione" che sta alla base della tutela costituzionale dei diritti fondamentali. La tutela contro le omissioni legislative si estende sia ai casi in cui il legislatore, pur legiferando, regola la materia in modo non adeguato, irragionevole o sproporzionato, sia allorché il legislatore riserva espressamente una disciplina a una determinata categoria di individui, escludendone altre: in questo caso, l'omissione parziale assume i connotati di un'esclusione discriminatoria (Fernández, 2009: 13).

Non essendo operante nel nostro ordinamento, l'istituto del ricorso diretto contro le omissioni del legislatore ${ }^{21}$, la Corte costituzionale sanziona l'omissione legislativa adottando le sentenze di incostituzionalità c.d. "di tipo additivo": in tal modo il giudice costituzionale sana autonomamente la lacuna dichiarando una disposizione incostituzionale (non per ciò che dispone in quanto non prevede una determinata norma la cui presenza è necessaria per rendere costituzionalmente legittima una disposizione di legge. Con tale operazione interpretativa la Corte introduce nell'ordinamento una norma mancante, necessaria per ripristinare la costituzionalità violata (Silvestri, 1981: 1684; Colapietro, 1990; Pinardi, 2007).

Sono state numerose le sentenze additive in materia penale, finalizzate soprattutto ad assicurare l'equità del processo e i diritti delle parti ${ }^{22}$, così come ha fatto ricorso a sentenze additive per risolvere questioni aventi a oggetto diritti di natura sociale, assistenziale e previdenziale. Nel rimuovere situazioni di diseguaglianza nella fruizione di prestazioni pubbliche la Corte si è trovato dinanzi a un'alternativa per assicurare il rispetto del principio di eguaglianza, potendo sia ampliare, quanto ridurre la platea dei beneficiari di determinate prestazioni: come è stato significativamente precisato, "l'operatività del principio di eguaglianza non è unidirezionalmente e necessariamente diretta ad

21 Si rinvia in proposito a Rolla, 2012: 168.

22 Ad esempio, il giudice costituzionale ha dichiarato costituzionalmente illegittima la disposizione che prevedeva la presenza del pubblico ministero all'interrogatorio dell'imputato senza che fosse assicurata anche presenza al medesimo interrogatorio del difensore dell'imputato (sentenza n. 190 del 1970); così come la posizione dell'imputato condannato che non ha avuto tempestiva ed effettiva conoscenza del provvedimento è stata equiparata a quella prevista dall'art.175 del c.p.p. per il pubblico ministero, le parti e i difensori (sentenza n. 317 del 2009). 
estendere la portata di una disciplina più favorevole..., ma può dispiegarsi anche nel senso di rimuovere l'ingiustificato privilegio di una disciplina più favorevole rispetto a quella indicata a comparazione"23.

Riflettendo sul trend giurisprudenziale della Corte costituzionale italiana a 63 anni dalla sua prima sentenza si nota una modificazione progressiva della sua collocazione all'interno del "parallelogramma" dei poteri costituzionali. Se, per un verso, in materia di diritti individuali e di libertà la natura di sindacato accentrato di costituzionalità entra in "competizione" con il concomitante attivismo delle giurisdizioni comuni e di quelle sovranazionali ${ }^{24}$; per un altro verso, nelle relazioni con gli altri "poteri" - come vedremo nei prossimi paragrafi- implementa la sua posizione di interprete (piuttosto che attuatore) della Costituzione, di garante dei principi costituzionali (in particolare di quello dell'unitarietà dell'ordinamento generale e della salvaguardia dell'intima dignità di tutti gli individui) e della ragionevolezza e proporzionalità degli interventi del legislatore.

\section{DUE CASI DI CONTROVERSO RAPPORTO TRA IL GIUDICE COSTITUZIONALE E IL PARLAMENTO}

Al fine di meglio evidenziare il complesso rapporto tra discrezionalità legislativa e controllo della legittimità costituzionale delle leggi può essere utile focalizzare l'attenzione su due situazioni in cui il giudice costituzionale ha manifestato una posizione "particolare" nei confronti delle scelte politiche del Parlamento: mi riferisco, per un verso, all'atteggiamento di sostanziale "deferenza" assunta allorché ha dovuto decidere su "questioni scientifiche controverse", che chiamano in causa le relazioni che intercorrono tra la scienza, la morale e il diritto (Veronesi, 2007; D’Amico, 2008; Chieffi, 1993; Carusi, 2011); per un altro verso, al tentativo della Corte di "affidare" al legislatore la soluzione di una questione di legittimità costituzionale che investe problematiche non strettamente giuridiche, nei confronti delle quali il giudizio sociale non appare univoco (Razzano, 2019).

Nel primo caso, il giudice costituzionale ha individuato - in generenel Parlamento il soggetto primario cui compete operare un bilanciamento (il c.d. definational balancing), limitandosi a verificare se lo stesso conteneva un ragionevole e proporzionato equilibrio tra gli interessi e i diritti in potenziale conflitto.

23 Ad esempio, nella sentenza n. 421 del 1995.

24 Si rinvia a Rolla, 2012: 181. 
Nel compiere tale operazione ha assunto, in genere, un atteggiamento di cautela e di circospezione, preoccupandosi di non contrapporre un propria "visione" della materia, alternativa rispetto a quella codificata nella legge: se possibile, non entra nel merito scientifico dei problemi coinvolti, preferendo valorizzare le ragioni schiettamente giuridiche ${ }^{25}$. Inoltre, come ha avuto occasione di precisare, dalla circostanza che una norma si fondi su di una base scientifica non certa non deriva necessariamente l'incostituzionalità della disciplina della cui legittimità si dubita ${ }^{26}$.

$\mathrm{Nel}$ fondare la ratio decidendi delle proprie decisioni, il giudice costituzionale parte, in genere, da una presunzione di legittimità delle scelte del Parlamento, ritenendo che queste possano essere ribaltate soltanto nel caso in cui i dati scientifici su cui la legge si fonda siano "incontrovertibilmente erronei o raggiungano una tale livello di indeterminatezza da non consentire in alcun modo un'interpretazione ed un'applicazione razionali da parte del giudice $^{27}$.

Siffatta presunzione, tuttavia, viene meno quando le norme mettono in discussione la salvaguardia di diritti di libertà tutelati dalla Costituzione: specialmente se il parametro del giudizio e rappresentato dal diritto all'autodeterminazione, alla salute, alla libertà di ricerca scientifica.

Importanti sono le argomentazioni con le quali il giudice costituzionale ha dichiarato l'incostituzionalità di alcune importanti norme della legge $\mathrm{n}$. 40 del 2004 in materia di procreazione medicalmente assistita, ritenute lesive dei principi di dignità, di autodeterminazione individuale e del diritto alla salute $^{28}$. Alcuni passi della motivazione sono, a nostro avviso, di particolare interesse: ad esempio, quando si afferma che la scelta di una coppia di diventare genitori "costituisce espressione della fondamentale e generale libertà di autodeterminarsi", per cui un divieto assoluto imposto al suo esercizio deve essere "ragionevolmente e congruamente giustificato dall'impossibilità di tutelare altrimenti interessi di pari rango" 29 . Sempre in materia medica, la Corte

25 Emblematica è, in proposito, l'ordinanza n. 369 del 2006, in materia di diagnosi preimpianto nelle procedure di procreazione medicalmente assistita, in cui la Corte perviene ad un'ordinanza di inammissibilità usando esclusivamente argomenti giuridico-formali.

26 Sentenza n. 185 del 1998.

27 Vedi, ad esempio, la sentenza n. 114 del 1998.

28 Tra i molti contributi, Casonato y Frosini, 2006.

29 Vedi ad esempio, la sentenza n. 162 del 2014. Inoltre, sempre secondo il giudice costituzionale, un divieto assoluto di ricorrere alle tecniche di procreazione medicalmente assistita da parte di coppie fertili portatrici di malattie genetiche trasmissibili lede il diritto costituzionale alla salute e risulta irragionevolmente contradditorio rispetto a 
costituzionale ha considerato la volontà del paziente — da esprimersi attraverso l'istituto del "consenso informato" - un limite invalicabile per la legge, dal momento che tale istituto è riconducibile a due diritti fondamentali della persona (la salute e l' autodeterminazione). Secondo le argomentazioni del giudice costituzionale "ogni individuo ha il diritto a essere curato e a ricevere le opportune informazioni in ordine alla natura e ai possibili sviluppi del percorso terapeutico" 30 -

In tema di libertà della ricerca scientifica, infine, la Corte ha dichiarato l'incostituzionalità di norme che invadevano l'autonomia professionale dello scienziato o del professionista, affermando che non spetta al legislatore "stabilire direttamente e specificamente quali siano le pratiche terapeutiche ammesse", dal momento che in materia "la regola di fondo è costituita dalla autonomia e dalla responsabilità del medico che, sempre con il consenso del paziente, opera le scelte professionali basandosi sullo stato delle conoscenze a disposizione" ${ }^{31}$. Mentre in un'altra decisione ha precisato che le valutazioni in tema di scelte terapeutiche non rientrano nella "pura discrezionalità politica del legislatore", il quale deve "tenere conto anche degli indirizzi fondati sulla verifica dello stato delle conoscenze scientifiche e delle evidenze sperimentali acquisite"32.

Il secondo caso cui abbiamo accennato all'inizio del paragrafo risulta - a sua volta - difficilmente inquadrabile all'interno dei tipi di tecniche interpretative richiamate nel paragrafo precedente. Si tratta dell'ordinanza n. 207 del 2018 con la quale la Corte affronta la questione di legittimità costituzionale dell'art.580 del codice penale che punisce l'istigazione o l'aiuto al suicidio: la sua peculiarità (od originalità) deriva dal fatto che il collegio giudicante, dopo aver rilevato che "l'attuale assetto normativo concernente il fine vita lascia prive di adeguata tutela determinate situazioni costituzionalmente meritevoli di protezione e da bilanciare con altri beni costituzionalmente rilevanti, non risolve il dubbio sollevato dal giudice a quo con una sentenza di accoglimento o con una sentenza "additiva" o "additiva di principio", ma decide di "non

quanto già da tempo previsto dalla legge n. 194 del 1978 (disciplina dell'aborto) che consente "l'interruzione volontaria (anche reiterata) di gravidanze naturali al fine di perseguire l'obiettivo di procreare un figlio non affetto dalla specifica patologia ereditaria” di cui la coppia è portatrice (sentenza n. 94 del 2015).

30 Vedi sentenza n. 438 del 2008

31 Vedi sentenza n. 282 del 2002.

32 Vedi sentenza n. 8 del 2011. Sempre in coerenza con siffatto indirizzo giurisprudenziale ha ribadito che la legge deve riconoscere "al medico la possibilità di una valutazione sulla base delle più aggiornate e accreditate conoscenze tecnico scientifiche" (sentenza n. 151 del 2009). 
decidere". Infatti rinvia all'udienza pubblica del 24 settembre 2019 la trattazione della questione di costituzionalità al fine di "consentire in primo luogo al Parlamento di intervenire con un'appropriata disciplina”.

Nel corpo della motivazione sembrano giustapporsi due filoni argomentativi distinti (che evidenziano, forse, una divisione all'interno del collegio). L'uno evidenzia in astratto la non contrarietà alla Costituzione della normativa che incrimina l'istigazione e l'aiuto al suicidio: sia perché dall'art. 2 Cost. "discende il dovere dello Stato di tutelare la vita di ogni individuo" anche creando attorno al soggetto interessato una sorta di "cintura protettiva" inibendo ai terzi di cooperare in qualsiasi modo con lui"; sia in quanto la norma penale sarebbe funzionale alla tutela del diritto alla vita delle persone più deboli e vulnerabili.

L'altro, per contro, prende le mosse dall'oggetto specifico del giudizio $a$ quo prendendo atto che possono determinarsi situazioni nelle quali l'assistenza di terzi nel porre fine alla sua vita può presentarsi al malato come l'unica via d'uscita per sottrarsi, nel rispetto del proprio concetto di dignità della persona, a un mantenimento artificiale in vita non più voluto e che egli ha il diritto di rifiutare in base all'art. 32, secondo comma, Cost.”. Di conseguenza, sempre ad avviso del giudice costituzionale "entro lo specifico ambito considerato, il divieto assoluto di aiuto al suicidio finisce, quindi, per limitare la libertà di autodeterminazione del malato nella scelta delle terapie, comprese quelle finalizzate a liberarlo dalle sofferenze, scaturente dagli artt. 2, 13 e 32, secondo comma, Cost." 33 .

L'argomentare della Corte sembra prefigurare - trattandosi di un giudizio incidentale, quindi condizionato da un necessario legame con la concretezza della questione a quo - una sentenza di tipo additivo, oppure (qualora il giudice costituzionale ritenga di impegnare il Parlamento ad approvare una disciplina organica della complessa tematica del "fine vita" e della dignità al momento della morte) di una additiva di principio.

Invece, la Corte con una ordinanza demanda la soluzione del problema al legislatore: il giudice sembra voler rinunciare a realizzare un "ad hoc balance" richiedendo al Parlamento un "definitional balance". I motivi di tale rinuncia appaio, a nostro avviso, difficilmente comprensibili e solo la viva vox dell'organo di giustizia costituzionale potrebbe chiarirli. In attesa, non si può sfuggire al sospetto che il comportamento della Corte possa essere stato determinato dall'intenzione non tanto di rivolgere un "monito" al Parlamento, quanto di evitare una scelta difficile e non condivisa dall'intero collegio ${ }^{34}$.

33 Così in ordinanza n. 207 del 2018.

34 Tra i molti interventi si rinvia a Razzano, 2019. 


\section{IL DIFFICILE EQUILIBRIO TRA GARANZIA DEI DIRITTI SOCIALI E VINCOLI ECONOMICO-FINANZIARI}

I diritti sociali costituiscono una partizione della categoria generale dei diritti dell'individuo, ma debbono anche essere annoverati tra gli elementi che concorrono a caratterizzare la forma di Stato democratica e sociale: tale duplicità fa sì essi, per un verso, arricchiscono il principio personalistico e, per un altro verso, rappresentano i principi orientatori che lo Stato deve perseguite. Alcune Costituzioni —ad esempio, gli ordinamenti di cultura anglosassone e la Spagna - valorizzano preferenzialmente questo secondo profilo; altre, invece - come quella italiana - li inseriscono a pieno titolo tra i diritti costituzionali dell'individuo.

In entrambi i casi, tuttavia, i diritti sociali sono espressione del dovere dello Stato di assicurare determinate prestazioni; tuttavia, l'ampiezza e la natura di tali prestazioni non costituiscono una "variabile indipendente", in quanto le scelte legislative sono condizionate da alcuni fattori, come la quantità delle risorse disponibili, la condizione complessiva della finanza pubblica e i vincoli imposti dall'adesione all'Unione europea: si può parlare in proposito di "diritti finanziariamente condizionati" 35 .

In altri termini, un medesimo diritto (all'abitazione, all'istruzione, ai servizi sociali, al lavoro e a un salario dignitoso, ecc.) può contenere al proprio interno un set di prestazioni più ampio in fasi economicamente espansive oppure più circoscritto (sino a ridursi al "nucleo indisponibile") in situazioni di difficoltà economiche o di crisi del sistema di finanza pubblica.

Di conseguenza, è difficile determinare in astratto il grado di effettività assicurato ai diritti sociali dalla giurisprudenza costituzionale, dal momento che in siffatta valutazione entra in gioco una molteplicità di elementi eterogenei come la struttura delle disposizioni costituzionali, il contesto economico e sociale in cui il giudice deve decidere, la sensibilità sociale del collegio giudicante. Inoltre, in questa materia il giudice costituzionale non deve decidere "a rime obbligate", bensì possiede un ampio margine di apprezzamento, il quale però trova le sue "colonne d'Ercole" non valicabili nella necessità che le disposizioni oggetto del giudizio siano coerenti con il principio di eguaglianza (ragionevolezza) e di proporzionalità e non intacchino il contenuto essenziale delle prestazioni da assicurare a tutti i cittadini. Come ha precisato la Corte costituzionale, l'effettiva fruibilità del nucleo indefettibile dei diritti spettanti a determinate categorie di individui (nel caso di specie si trattava di persone con disabilità) non può dipendere da scelte finanziarie che il legislatore com-

35 Così: Merusi, 1990: 30. 
pie con previsioni che lasciano incerta nell' an e nel quantum la misura della contribuzione, pena violare la dignità insita in ogni individuo ${ }^{36}$.

Tuttavia, esiste una diversità di atteggiamento tra le diverse giurisdizioni costituzionali: tra chi affida valutazione delle scelte del legislatore al rispetto rigoroso di determinati test di interpretazione ${ }^{37}$, e chi agisce pragmaticamente, valutando caso per caso — come nel caso della Corte costituzionale italiana-: nella sua ricca giurisprudenza non emerge un leading case, né le motivazioni delle sue sentenze scaturiscono da procedimenti logici, da step argomentativi predeterminati ed esplicitati nelle sue rationes decidendi. Piuttosto, nelle singole questioni sottoposte al suo giudizio, sembra ricercare un ragionevole punto di equilibrio tra la tutela dei diritti sociali e le compatibilità economiche e finanziarie contenute nelle scelte di bilancio, tra le aspettative sociali e i vincoli economici dai quali il Parlamento non può prescindere.

In diverse occasioni, la Corte costituzionale ha precisato che "il legislatore nel determinare l'ammontare delle prestazioni sociali può tener conto della disponibilità delle risorse finanziarie purché le sue scelte siano ragionevoli e rispettose del principio di proporzionalità"38; così come ha considerato ammissibili contributi di solidarietà a carico delle pensioni di importo più elevato qualora trovino fondamento in una crisi contingente e grave del sistema previdenziale ${ }^{39}$.

Spesso il giudice costituzionale — pur salvaguardando la sostanza dei diversi diritti sociali- ha assunto un atteggiamento di cautela, intraprendendo un percorso giurisprudenziale attento a non invadere" il terreno" riservato alla discrezionalità politica del Parlamento (Bianchi, 2006; Salazar, 2000; Furno, 2017). Nei periodi di "austerità" e di crisi delle finanze pubbliche, come è stato evidenziato dalla dottrina, la sua giurisprudenza non è apparsa cristallina, rimanendo incertezze circa l'effettiva quantità di sacrifici che possono essere

36 Si vedano, le sentenze n. 275 del 2016 e n. 83 del 2019.

37 Ad esempio, il Tribunale costituzionale federale di Germania ha individuato un test articolato essenzialmente su tre verifiche inerenti al rispetto del principio di effettività, di indispensabilità (verificare l'esistenza di altre misure che possono rendere sufficiente la protezione), di razionalità. Mentre il Tribunale costituzionale del Portogallo è andato oltre sino a precisare che in ossequio al principio di eguaglianza il peso dei sacrifici deve essere ripartito tra le diverse categorie di cittadini in modo equilibrato e motivato, dimostrando di aver esplorato la fattibilità di misure alternative rispetto a quelle prescelte).

38 Ad esempio, nelle sentenze n. 180 del1982, n. 220 del 1988, n. 73 del 1992, n. 485 del 1992 e n. 347 del 1997.

39 Sentenza n. 173 del 2016. Vedi: Salazar, 2015: 153; D'Amico y Biondi, 2017; Gambino, 2015; Busata, 2018: 83; Brancati, 2018; Ciolli, 2012. 
patiti da differenti categorie di persone a cause di esigenze di politica economica o di vincoli posti dalle istituzioni comunitarie. ${ }^{40}$ In altre situazioni, la Corte costituzionale ha tenuto in particolare considerazione le conseguenze finanziarie delle sue pronunce: sia facendo precedere le decisioni da un'attività istruttoria finalizzata a quantificare gli oneri finanziari conseguenti a un'eventuale decisione di accoglimento, sia ricorrendo a sentenze additive di principio che le consentono di affermare la lesione di un diritto, demandando al legislatore la scelta dei modi con cui conseguire la copertura dei relativi costi. In tal modo, si realizza una "distribuzione del lavoro" tra il giudice costituzionale — che indica il principio a cui rifarsi per sopperire al vizio di incostituzionalità- $\mathrm{e}$ il legislatore che deve dare seguito al principio individuando le modalità e le relative coperture ${ }^{41}$.

In altre decisioni la Corte ha riconosciuto la discrezionalità del legislatore nel modulare il contenuto dei diritti sociali, limitandosi a verificare che la loro configurazione legale non leda in modo irragionevole il contenuto essenziale del diritto: in altri termini, ammette una gradualità nell'erogazione di prestazioni sociali e riconosce che le esigenze finanziarie possano limitare l'ampiezza delle prestazioni sociali, purché non si vanifichi il contenuto essenziale del diritto ${ }^{42}$.

Infine, ha individuato nel principio di dignità (che garantisce tutti gli individui indipendentemente dalla loro traiettoria di vita e dallo status civitatis) il confine oltre il quale la discrezionalità legislativa non può spingersi (Ceccherini, 2008). A tal fine, si può richiamare l'orientamento del giudice costituzionale secondo cui alcune cure ospedaliere e ambulatoriali che costituiscono il nucleo essenziale del diritto alla salute devono essere erogate a favore di tutti, anche "indipendentemente dalla regolarità della posizione delle persone che ne beneficiano ${ }^{43}$. Mentre in tema di diritto allo studio dei disabili ha precisato che "il nucleo di garanzie minime per renderlo effettivo deve essere assicurato al di là di ogni esigenza di bilancio" 44 .

40 Così, ad esempio, Guiglia, 2018.

41 Ad esempio, sentenza n. 243 del 1993.

42 Nella sentenza n. 222 del 2013 il giudice costituzionale ha precisato che l'individuazione dei beneficiari di alcune prestazioni sociali può essere circoscritta in ragione della limitatezza delle risorse disponibili, ma tale scelta deve essere operata sempre e comunque in ossequio al principio di ragionevolezza.

43 Così nella sentenza n. 299 del 2010 (Biondi, 2013).

44 Sentenza n. 275 del 2016. 


\section{LA CORTE COSTITUZIONALE COME GARANTE DELL' UNITÀ DELL'ORDINAMENTO E ARBITRA NEI RAPPORTI TRA LO STATO E LE REGIONI}

I costituenti italiani, allorché affrontarono il tema della forma di Stato sotto il profilo della distribuzione territoriale dei processi di decisione politica, furono condizionati nella loro scelta da due elementi: che le esperienze storiche a cui ispirarsi erano costituite da due prototipi contrapposti rappresentati dagli Stati federali e gli ordinamenti unitari centralizzati; che non si poteva del tutto prescindere dal processo storico che aveva portato alla nascita dello Stato italiano e, successivamente, alla sua trasformazione e involuzione durante il periodo fascista ${ }^{45}$.

Di conseguenza, il punto di sintesi tra le differenti posizioni fu trovato in una forma di Stato "intermedia" tra centralismo e federalismo, formalizzata nell'approvazione di un ordine del giorno con cui la seconda Sottocommissione definì i confini entro cui si sarebbero dovute incanalare le scelte dei costituenti: si intendeva, cioè, istituire le Regioni come enti autarchici (capaci di perseguire fini propri), autonomi (forniti di autonomia organizzativa e di potestà legislativa nelle materie di propria competenza), rappresentativi (dotati di organi elettivi a suffragio universale) e con autonomia finanziaria (Baschieri et al., 1949: 380).

Nel contrasto tra centralisti, regionalisti "spinti" e "tiepidi" prevalse una posizione mediana che, conformemente alla natura tipicamente pattizia della Costituzione italiana, evitò — per quanto possibile - formulazioni che fossero espressione omogenea di una visione unilaterale dello Stato. Infatti, la particolarità del regionalismo italiano che emerge dalle disposizioni costituzionali del Titolo $\mathrm{V}$ poggia su tre pilastri.

Innanzitutto, la Costituzione repubblicana introduce un elemento di discontinuità nell'evoluzione della forma di Stato, affermando per la prima volta la piena compatibilità tra natura unitaria dello Stato e decentramento politico (Esposito, 1954: 82).

In secondo luogo, è molto forte l' attenzione per le esigenze unitarie dell'ordinamento, che vengono salvaguardate sia premurandosi di incardinare il sistema delle autonomie territoriali all'interno di un contesto istituzionale attento alla cura degli interessi nazionali, sia attribuendo allo Stato competenze specifiche affinché le differenze tra le diverse comunità regionali non intacchino la parità tra i cittadini nel godimento dei diritti fondamentali e il principio di solidarietà. A differenza della regionalizzazione introdotta in Spagna (Rolla,

45 Si veda Ghisalberti, 2000. Con riferimento al fascismo, Melis, 2018. 
2008: 149), l'autonomia non tanto scaturisce dal principio dispositivo, ma si sviluppa su impulso dell'attività normativa dello Stato: come emerge dalla formulazione dell'art. 5 Cost. si utilizza l'espressione "promuove" per evidenziare che il processo di decentramento scaturisce dall'azione unilaterale dello Stato, che viene individuato come il soggetto che conforma l'autonomia delle comunità territoriali.

Infine, la Costituzione ha istituzionalizzato un sistema rigido di relazioni tra lo Stato e le Regioni, ispirato a una visione garantistica dell'autonomia, preoccupata di separare le rispettive attribuzioni e di prevedere meccanismi politici e giurisdizionali a tutela della rispettiva sfera di competenza: come è stato efficacemente rilevato, dal testo costituzionale emerge la presenza di livelli istituzionali che "si confrontano e si contrappongono restando arroccati all'interno di ambiti di competenza distinti e separati” (Bartole, 1985: 28). Di conseguenza, nel testo costituzionale manca l'individuazione di strumenti di collaborazione tra interistituzionale; mentre si è tentato di introdurre una demarcazione rigida tra le competenze dei diversi livelli istituzionali, individuando nella Corte costituzionale l'arbitro cui rivolgersi per risolvere e conflitti di competenza.

Sin dai primi anni di funzionamento delle Regioni la dottrina si divise circa la posizione che il giudice costituzionale avrebbe assunto all'interno del contenzioso tra lo Stato e le Regioni: tra chi era propenso a prevedere che avrebbe adottato indirizzi giurisprudenziali rigorosi nella salvaguardia delle prerogative costituzionali delle Regioni e chi, per contro, era dell'avviso che l'organo di giustizia costituzionale si sarebbe limitato a confermare nella sostanza le soluzione adottate dallo Stato ovvero "concordate" tra i livelli istituzionali, mettendo al loro servizio "il più o meno spiccato acume esegetico dei giudici estensori delle sentenze" (Bassanini, 1978: 219; Paladini, 1979: 273). In realtà, se l'attenzione si concentra sugli esiti complessivi della giurisprudenza costituzionale è possibile intravedere nel comportamento della Corte una posizione articolata, difficilmente inquadrabile in schemi precostituiti.

Nella fase iniziale, di avvio dell'esperienza regionale, la Corte costituzionale ha contribuito a mettere a fuoco le caratteristiche principali del sistema, svolgendo una preziosa attività di supplenza innanzi ad alcune "lacune" del testo costituzionale. Alcuni istituti e principi oggi consolidati sono stati il frutto della sua attività interpretativa, piuttosto che della legislazione di attuazione del testo costituzionale: è il caso, ad esempio, della funzione di indirizzo e di coordinamento, della disciplina delle attività regionali di rilievo internazionale, dei criteri per determinare il concetto legale di materia regionale, della definizione dei rapporti tra la legislazione statale di principio e le leggi 
regionali nelle materie di competenza concorrente, dell'indicazione delle relazioni tra le Regioni e l'Unione europea ${ }^{46}$.

L'azione di supplenza della Corte è stata significativa anche in sede di attuazione della legge di revisione costituzionale n. 3 del 2001, allorché ha dovuto risolvere diverse problematiche conseguenti alla lacunosa formulazione del testo di legge. Non va sottovalutato, in proposito, che la Corte, a causa della mancata inserzione nella legge costituzionale n. 3 del 2001 di norme finali e transitorie, si è fatta carico, in più occasioni, dell'esigenza di assicurare la continuità e la funzionalità dei processi istituzionali, nonché di garantire la certezza del diritto e la sicurezza giuridica. La sua giurisprudenza in merito evidenzia un atteggiamento di cautela e la preoccupazione di assicurare una ragionevole continuità nel funzionamento del sistema costituzionale (Rolla, 2007).

D'altra parte, siffatto atteggiamento appare comprensibile, dal momento che il giudice di costituzionalità delle leggi ha dovuto navigare tra Scilla e Cariddi, tra il rischio di favorire la continuità con il passato (attraverso un' interpretazione restrittiva delle disposizioni costituzionali) e il pericolo di attuare in via giurisdizionale - $\mathrm{a}$ fronte dell'inerzia del legislatore- le disposizioni del "nuovo" titolo V della Costituzione.

In tema di finanza pubblica il giudice costituzionale ha avvertito l'esigenza che il passaggio dal vecchio al nuovo sistema avvenisse in modo graduale e ha auspicato la definizione di una disciplina transitoria che consenta l'ordinato passaggio dal vecchio al nuovo sistema, che poteva decorrere soltanto successivamente alla definizione da parte dello Stato di "un complessivo ridisegno dell'autonomia tributaria delle Regioni”" ${ }^{77}$. Egualmente, ha subordinato l'operatività delle nuove competenze legislative delle Regioni all'effettiva operatività delle strutture e di servizi regionali, ritenendo prioritario garantire la continuità delle funzioni pubbliche.

Secondo questa prospettiva, il giudice costituzionale ha riconosciuto, ad esempio, transitoriamente la validità degli atti di programmazione della rete scolastica compiuti dal Ministero, in attesa che le Regioni provvedano a dotarsi di apparati burocratici regionali preposti all'esercizio di tale funzione. Mentre in materia di attività culturali, ha negato che l'entrata in vigore della legge

46 In effetti, la giurisprudenza della Corte costituzionale in materia di Regioni è stata ampiamente studiata e dibattuta dalla dottrina, sino al punto che anche in Italia - come è stato detto in Spagna - si può parlare di diritto regionale giurisprudenziale (Aragón, 1986: 7).

47 Si vedano, ad esempio, la sentenza n. 37 del 2004 e la sentenza n. 31 del 2004. 
costituzionale n. 3 del 2001 abbia determinato l'automatica sopravvenuta incostituzionalità della legislazione statale vigente ${ }^{48}$.

Il giudice costituzionale non si è limitato a svolgere un'attività di supplenza del legislatore ordinario, ma ha altresì individuato, per un verso, nello Stato il principale soggetto attuatore dell'autonomia regionale ed assunto, per un altro verso, il ruolo di custode del carattere unitario dell'ordinamento.

In relazione al primo profilo, la Corte ha affidato alla legislazione statale il compito di determinare il significato normativo delle materie regionali, introducendo una sostanziale decostituzionalizzazione degli ambiti di competenza a regionale, dal momento che questi variavano a seconda della concreta definizione giuridica delle materie indotta dall'evolversi della produzione normativa ${ }^{49}$. Così come, nel caso della competenza legislativa concorrente, ha affermato che i principi delle materie che fungono da limite alla potestà legislativa regionale debbono essere ricavati —in assenza di apposite leggi di principio - in via interpretativa sulla base della legislazione statale vigente. Inoltre, ha ripetutamente affermato che la competenza delle Regioni in una determinata materia non è astratta, ma diviene effettivamente rivendicabile nel momento in cui la esercita concretamente: di conseguenza, lo Stato ha potuto legiferare in materie di spettanza regionale a fronte di un'inerzia da parte delle singole Regioni.

Ma l'elemento che maggiormente ha caratterizzato l'intera giurisprudenza costituzionale in materia di rapporti tra lo Stato e le Regioni è costituito dalla sua attenzione per la salvaguardia del principio unitario riconducibile all'art. 5 Cost. Tale principio è stato assimilato alla nozione di interesse nazionale, sia legittimando —all'interno di materie di competenza regionale- la riserva allo Stato di poteri necessari per la salvaguardia dell'unità giuridica o delle attività internazionali della Repubblica; sia attribuendo al Governo strumenti per esercitare l'attività di indirizzo e di coordinamento nei confronti dell'azione amministrativa regionale. Più recentemente, in una fase di crisi delle finanze pubbliche, il richiamo all'interesse nazionale ha consentito di giustificare l'espansione del legislatore statale in ambiti di spettanza regionale, al fine di evitare che la capacità autonoma di spesa delle Regioni impedisca la realizzazione degli obiettivi di finanza pubblica e il rispetto dei vincoli europei (Masala, 2016: 189).

Nel medesimo tempo la Corte costituzionale ha cercato di riequilibrare la garanzia degli interessi unitari dell'ordinamento "risarcendo" le Regioni del

48 Vedi sentenza n. 255 del 2004.

49 In merito al problema della definizione giuridica delle materia, si veda: Paladin, 1971: 3; Rolla, 1982: 100; D’Atena, 1974; Mangiameli, 1992. 
necessario rafforzamento del ruolo di indirizzo del legislatore statale attraverso l'introduzione del principio di "leale collaborazione" tra i livelli istituzionali. Se nel periodo di avvio dell'esperienza regionale, l'attenzione principale si è concentrata sulla determinazione dei criteri utilizzabili per individuare le materie di rispettiva competenza e sul perfezionamento dei meccanismi giurisdizionali utilizzabili per risolvere i possibili conflitti; per contro, in una fase di regionalismo maturo si delinea una inversione di tendenza a favore di un autonomismo di tipo cooperativo, rafforzando gli strumenti di partecipazione e di negoziazione (Tnoniatti, 2003: 167; Rolla, 2002: 321).

La giurisprudenza costituzionale ha qualificato la collaborazione come un "criterio" a cui occorre ispirarsi per rispettare il criterio di sussidiarietà nell'esercizio delle competenze ripartite tra lo Stato e le Regioni. A tal fine ha introdotto alcune regole per individuare le fattispecie in cui la leale collaborazione è necessaria: è il caso, ad esempio, in cui si deve decidere su fattispecie che coinvolgono materie tanto di competenza statale che regionale ("sovrapposizione di materie");oppure quando una determinata attività, pur formalmente rientrante nella potestà normativa regionale, viene avocata dallo Stato per esigenze di natura unitaria, in base al criterio di sussidiarietà ${ }^{50}$. Inoltre, il giudice costituzionale ha precisato che la collaborazione deve essere sostanziale, nel senso che il procedimento partecipativo avvenga con modalità tali che rivelino un effettivo animus cooperativo ${ }^{51}$.

Va, tuttavia, precisato che sinora la Corte ha limitato l'operatività della leale collaborazione alle fonti di grado secondario e amministrative, escludendola nei confronti delle leggi, a meno che tali procedure non siano, direttamente o indirettamente, previste dalla Costituzione. Tale orientamento restrittivo non considera, tuttavia, che la garanzia istituzionale del principio di autonomia dovrebbe operare anche nei confronti del procedimento legislativo: infatti, la previsione dell'art. 70 Cost. (La funzione legislativa è esercitata collettivamente dalle due Camere) deve essere interpretata in stretto collegamento con il principio generale affermato dall'art. 5 Cost., secondo cui la Repubblica deve adeguare i metodi della legislazione alle esigenze dell'autonomia e del decentramento.

Va, quindi, positivamente valutata una prima, timida apertura alla leale collaborazione nei procedimenti legislativi, che sembra emergere da un obiter dictum contenuto nella sentenza n. 251 del $2016^{52}$.

50 Ad esempio, le sentenze n. 330 del 2011 e n. 109 del 2011,

51 Ad esempio nella sentenza n. 255 del 2011.

52 In questa sentenza il giudice costituzionale, a proposito di alcuni decreti legislativi che incidevano su materie di competenza legislativa "mista" ha precisato che "occorre 


\section{LA GIUSTIZIA COSTITUZIONALE DINANZI ALLE ASPIRAZIONI IDENTITARIE DI ALCUNI TERRITORI}

L' attenzione per la natura unitaria dell'ordinamento si è manifestata in modo evidente nelle questioni in cui la Corte ha affrontato rivendicazioni di natura identitaria delle Regioni. In generale, si può affermare che la salvaguardia delle esigenze unitarie e la tutela degli interessi generali hanno caratterizzato una giurisprudenza favorevole ad assicurare l'omogeneità del sistema delle autonomie regionali e a depotenziare le spinte alla differenziazione territoriale.

A tal proposito, non va sottovalutato come un'idea delle Regioni quali enti di natura amministrativa (piuttosto che costituzionale) abbia trovato un conforto culturale nella dottrina amministrativa, cui si debbono i primi inquadramenti teorici della natura degli enti territoriali (Romano, 1946: 15; Zanobini, 1950: 182; Esposito, 1954: 6; Martines, 1952: 101; Berti, 1975: 277; Giannini, 1951: 878). Mi riferisco, in particolare, per un verso, alla concezione unitaria dell'amministrazione, considerata un corpo omogeneamente rivolto alla specificazione della volontà generale del legislatore, i cui atti debbono essere conformi alla volontà generale dello Stato, non potendosi ad essa contrapporre ${ }^{53}$; per un altro verso, all'idea che non si possano configurare poteri autonomi dalla legge - per cui lo status di autonomia può essere riconosciuto soltanto entro i confini e secondo i modi e le regole determinati dalla legge (una sorta di condizione di "libertà nell'ambito della legge").

La giurisprudenza costituzionale sembra aver rafforzato con coerenza l'idea di un regionalismo non solo unitario, ma anche uniforme e tale visione ha potuto giovarsi del fatto che in materia di interpretazione del Titolo V della Costituzione la Corte non si è trovata nella necessità di decidere "a rime obbligate", dato che — come è stato convincentemente affermato- "si va sempre di più affermando la tendenza a ravvisare nelle decisioni dei giudici continentali, e con essi di quelli del nostro Paese, il portato non già di una mera traduzione in termini concreti della volontà legislativa, ma il risultato di

contemperare le ragioni dell'esercizio unitario delle stesse con la garanzia delle funzioni costituzionalmente attribuite alle autonomie"; e che tale obiettivo può essere conseguito attraverso una necessaria "intesa" tra i livelli istituzionali da raggiungere in sede di Conferenza unificata tra lo Stato e le Regioni. In proposito, Bifulco, 2017.

53 Da ciò il consenso per quelle teorie le quali sostenevano che gli interessi perseguiti dagli enti dotati di autonomia, pur possedendo un autonomo riconoscimento, dovevano sempre "subordinarsi a quelli generali che, per estensione, per contenuto e per grado è compito dello Stato di far valere"; ovvero ritenevano che fossero autonomi gli enti che "col provvedere a fini propri, provvedono a fini che sono anche dello Stato.... che lo Stato ritiene di attuare meglio attraverso l'opera di enti". 
un processo più complesso, nel quale si inseriscono scelte e valutazioni autonome degli stessi giudici” (Bartole, 1980: 9).

Siffatto orientamento si è manifestato in diverse occasioni.

Ciò è avvenuto, innanzitutto, nelle questioni in cui ha dovuto misurarsi con la specialità delle cinque Regioni ad autonomia differenziata. Gli orientamenti manifestati in seno all'assemblea costituente erano sufficientemente espliciti nel ritenere che alle Regioni a autonomia speciale dovesse essere attribuita una posizione di maggior rilievo, sia sotto il profilo quantitativo che qualitativo: anche se appare problematico precisare le competenze in cui si sostanzia "quel di più" che deve essere loro riconosciuto, era indubbio che la specialità dovesse implicare un'autonomia più vasta e un complesso di attribuzioni più intense, non potendo lo status di specialità "tradursi nella privazione, anche parziale, di taluni poteri o funzioni garantiti alla regioni dalla Costituzione" (Bassanini, 1971: 1732; Azzena, 1979). Inoltre, nelle impostazioni iniziali si riteneva che la specialità dovesse differenziare ciascuna delle Regioni elencate nell'art. 116 Cost. dalle altre.

Tuttavia, il processo attuativo delle regioni ad autonomia speciale ha progressivamente depotenziato la specificità dei singoli ordinamenti, al punto che si può parlare di un "diritto comune" delle Regioni ad autonomia speciale; mentre nel caso dei rapporti con le regioni a statuto ordinario si è determinato - a giudizio di un'autorevole dottrina- un ribaltamento del modello risultante dalla Costituzione (D'Atena, 1991: 381). Tra i fattori che hanno concorso a svolgere tale funzione omogeneizzatrice un ruolo importante è stato assolto dalla giurisprudenza della Corte costituzionale che, sin dalle sue prime sentenze, ha prodotto una giurisprudenza finalizzata a omologare le diverse Regioni speciali e ad estendere a loro limiti formalmente previsti per le sole Regioni ordinarie (Bartole y Vandelli, 1980; Paladin, 1985: 77; Rolla, 1973: 1680). In altri termini, si sono stemperate — nel nome dell'unità dell'ordinamento e della priorità degli interessi nazionali- le diversità e appiattite le peculiarità dei singoli ordinamenti.

Interessante è la posizione assunta, successivamente, dalla Corte in merito alle richieste di modifica territoriale che coinvolgono Regioni ad autonomia speciale. Ad esempio, risolvendo un conflitto di attribuzione sollevato dalla Regione Valle d'Aosta nei confronti dello Stato, in relazione al procedimento di distacco di un Comune da una Regione ordinaria con conseguente aggregazione a una Regione speciale, il giudice costituzionale segue un'argomentazione meramente formale, mentre tralascia ogni riferimento al dato sostanziale sotteso al conflitto. Infatti, il giudice costituzionale, respingendo il ricorso, precisa, da un lato, che l'obbligo di coinvolgere gli enti interessati nel procedimento per la modificazione del territorio della Regione riguarda tutte le Regioni ordinarie ai sensi dell'art. 132 Cost.; dall'altro lato, che l'esito 
del referendum non produce effetti vincolanti nei confronti dell'altro ente interessato al procedimento di aggregazione ${ }^{54}$.

Così argomentando, la Corte non entra nel merito del problema procedurale sintetizzabile nel quesito come sia possibile modificare il territorio di una regione ad autonomia speciale. Infatti, se il $s u b$ procedimento interessante la Regione ordinaria è dettagliatamente disciplinato dall'art. 132 Cost., gli Statuti delle Regioni ad autonomia speciale non prevedono apposite procedure di modificazione e tale "omissione" induce a ritenere che i legislatori costituzionali non avessero preso in considerazione la possibilità di modificare il loro territorio ${ }^{55}$.

Il giudice costituzionale motiva - a nostro avviso- la decisione in modo volutamente lacunoso, in quanto non prende in considerazione (con opportuni obiter dicta) come si possa avviare e concludere il sub procedimento interno alle Regioni ad autonomia speciale, né contiene alcun indirizzo al legislatore su come disciplinare tale fase: alimentando, di fatto, un'incertezza circa il procedimento da adottare nel caso oggetto del conflitto di attribuzione $\mathrm{e}$ in ulteriori richieste interessanti altre Regioni ad autonomia speciale ${ }^{56}$.

L'ordinamento italiano non è riuscito a emanciparsi dall'idea che le Regioni siano espressione di un decentramento essenzialmente regolato "dall'alto" e territorialmente omogeneo sotto il profilo delle competenze. Le tappe che ne hanno scandito la nascita e il consolidamento evidenziano l'affermazione di una spinta centripeta finalizzata sia a assicurare l'uniformità dell'ordinamento generale attraverso l'attuazione di un "modello" comune che plasma omogeneamente l'organizzazione delle singole comunità territoriali; sia a neutralizzare le specificità territoriali attraverso l'attribuzione di comuni ambiti di autonomia e di competenza.

Tale visione dell'autonomia regionale, tuttavia, sembra oggi messa in crisi da un rafforzamento delle tendenze favorevoli a un regionalismo asimmetrico e di natura identitaria, in cui l'autonomia si lega alla "storia" dei territori e il rapporto con la tradizione si manifesta con il riconoscimento di specifici

54 Vedi, sentenza n. 66 del 2007.

55 Anzi, sembra che gli Statuti speciali abbiano inteso "pietrificare" l'individuazione del territorio al momento della loro istituzione D'altra parte, l'identificazione dei Comuni e delle Province appartenenti ad una Regione ad autonomia speciale, in alcuni casi, predeterminata dal dato geografico (come nel caso delle due isole), in altri dal fattore identitario e linguistico (come nel caso delle tre Regioni di confine).

56 Come nel caso dei tentativi di variazione territoriale ex art. 132 Cost. da parte di alcuni Comuni veneti confinanti con il Trentino-Alto Adige/Südtirol o il Friuli-Venezia Giulia, spinti anche dall'ambizione di accedere alle più favorevoli condizioni di autonomia finanziaria di cui beneficiano le due Regioni speciali. 
istituti o tradizioni giuridiche derogatorie rispetto all'ordinamento giuridico generale (Rolla, 2019: 73). Sono numerosi nel diritto comparato i casi in cui il riconoscimento di particolari forme di differenziazione è favorito dalla volontà di tutelare o rafforzare determinate minoranze; d'altra parte, un'autorevole dottrina ha ritenuto che sia l' elemento culturale il tratto giustificante il principio di autonomia (Haberle, 1994: 129), e che questo ultimo costituisca un criterio di organizzazione che favorisce la creazione di una comunità nazionale formata da una pluralità di comunità più circoscritte che mantengono la propria peculiarità (Fossas y Requejo, 1999).

Latteggiamento del giudice costituzionale italiano nei confronti delle tendenze identitarie di vario tipo può essere sinteticamente riassunto nella volontà di scongiurare che esse costituiscano un pericolo per il tessuto culturale unitario della Repubblica. Un esempio - a mio avviso - probante di siffatta preoccupazione può essere offerto da alcune decisioni inerenti la competenza delle Regioni di tutelare, valorizzare e promuovere con apposite leggi i gruppi linguistici minoritari presenti nei rispettivi territori.

Tra queste possiede un significato emblematico la sentenza n. 159 del 2009 con la quale la Corte costituzionale ha dichiarato l'illegittimità costituzionale della legge n. 29 del 2007 della Regione Friuli-Venezia Giulia contenente norme per la tutela, valorizzazione e promozione della lingua friulana. Indubbiamente alcune sue disposizioni costituivano un "evidente" forzatura del dettato costituzionale: ad esempio, la normativa sulla toponomastica, prevedendo l'utilizzazione della sola lingua minoritaria, sembrava contrastare con il riconoscimento della lingua italiana come lingua ufficiale; così come la scelta di estendere l'utilizzo obbligatorio della lingua friulana sull'intero territorio regionale -invece di circoscriverlo alle sole aree di insediamento del gruppo linguistico- appariva irragionevole e sproporzionata.

Si può, pertanto, comprendere che la Corte costituzionale possa aver manifestato preoccupazione sia per le conseguenze discriminatorie che la normativa poteva generare all'interno del territorio regionale nei confronti di coloro che non appartenevano alla minoranza linguistica, sia per gli effetti emulativi che una siffatta interpretazione delle competenze linguistiche regionali avrebbe potuto esercitare nei confronti di altre Regioni, sia speciali che ordinarie.

Tuttavia, il percorso argomentativo utilizzato dalla Corte costituzionale sembra comprimere oltre misura la competenza legislativa delle Regioni speciali in materia di tutela e valorizzazione delle minoranze linguistiche: con la sua giurisprudenza il giudice costituzionale ha depotenziato un principio qualificante la specialità regionale al fine di scongiurare il pericolo di una lesione del tessuto culturale unitario della Repubblica. Il giudice costituzionale, infatti, basa la sua ratio decidendi non già sugli Statuti delle regioni ad autonomia speciale, bensì sull'art. 6 Cost. (e la relativa legislazione di attuazione) che 
riserva allo Stato la competenza a individuare e disciplinare le lingue minoritarie Di conseguenza, la l Corte ha ridotto la competenza regionale in materia al rango di normativa di attuazione della legislazione statale.

Tale orientamento è stato più esplicitamente confermato in occasione di un ricorso avverso la legge n. 11 del 2009 della Regione Piemonte, allorché ha affermato che la cura del pluralismo linguistico deve essere riservata in prima istanza allo Stato, mentre le Regioni possono legiferare in materia di minoranze linguistiche e culturali presenti sul territorio purché in attuazione della disciplina statale ${ }^{57}$. Un'argomentazione che, a nostro avviso, lascia perplessi per un duplice motivo: in primo luogo, per la volontà di uniformare la competenza delle Regioni speciali a quella delle Regioni ordinarie proprio in una materia che è alla base della natura "speciale" di alcune Regioni; in secondo luogo, perché il giudice costituzionale ha avvallato nella fattispecie un'interpretazione restrittiva dell'espressione "Repubblica" presente nell'art. 6 Cost. ${ }^{58}$, intendendola come sinonimo dello Stato (Rolla y Ceccherini, 2004; Pizzorusso, 1967; Bartole et al., 1998; Palici, 2002; Poggeschi, 2010; Palermo y Woelk, 2008).

Da ultimo, la funzione del giudice costituzionale di garante dell'unità istituzionale e politica della Repubblica si è manifestata attraverso la posizione di decisa intransigenza con cui ha contrastato spinte e pulsioni di natura indipendentistica. A differenza del Tribunale costituzionale spagnolo — che, pur dichiarando con nettezza l'incostituzionalità del processo indipendentista catalano, ha ritenuto in un obiter dictum che l'acquisizione di uno status di indipendenza rappresenta un'aspirazione politica da perseguire nei modi e nelle forme regolate dalla Costituzione $-{ }^{59}$ il Tribunale costituzionale italiano ha affermato in diverse occasioni che una rivendicazione di indipendenza in ordinamenti retti da una Costituzione scritta e rigida sia "estranea" alla legalità costituzionale, dal momento che comporterebbe l'attribuzione alla Regione di tratti ordinamentali appartenenti a Stati federali.

Affrontando il tema della revisione dello Statuto speciale della Sardegna, la Corte costituzionale ha dichiarato l'illegittimità costituzionale della legge regionale n. 7 del 2007 (istitutiva di una "Consulta per il nuovo Statuto di autonomia e sovranità del popolo sardo") relativamente al punto in cui affidava alla Consulta regionale il compito di definire, oltre ai principi e i caratteri della identità regionale, le "ragioni fondanti l'autonomia e sovranità"

57 Si veda la sentenza n. 170 del 2010.

58 "La Repubblica tutela con apposite norme le minoranze linguistiche".

59 Sentenza n. 42 del 2014. Per maggiori riferimenti dottrinali sul punto si rinvia a Rolla, 2019: 685 . 
dell'isola. Il giudice della costituzionalità delle leggi intravede nelle disposizioni regionali il tentativo di delineare uno Statuto profondamente differenziato da quello attuale e "caratterizzato da istituti adeguati ad accentuati modelli di tipo federalistico, normalmente frutto di processi storici nei quali le entità territoriali componenti lo Stato federale mantengono forme ed istituti che risentono della loro preesistente condizione di sovranità" ${ }^{\circ 0}$.

Anche nel caso della legge n. 16 del 2014 della Regione Veneto avente ad oggetto l'indizione di un referendum consultivo sull'indipendenza del Veneto il giudice costituzionale giudice ha richiamato una costante giurisprudenza secondo cui "pluralismo e autonomia non consentono alle Regioni di qualificarsi in termini di sovranità, né permettono che i loro organi di governo siano assimilati a quelli dotati di rappresentanza nazionale" ${ }^{61}$. Infatti, a suo avviso, il referendum consultivo previsto dall'art. 1 della legge non solo riguarda scelte fondamentali di livello costituzionale, come tali precluse ai referendum regionali, "ma suggerisce sovvertimenti istituzionali radicalmente incompatibili con i fondamentali principi di unità e indivisibilità della Repubblica, di cui all'art. 5 Cost."; aggiunge inoltre che "l'unità della Repubblica è uno di quegli elementi così essenziali dell'ordinamento costituzionale da essere sottratti persino al potere di revisione costituzionale (sentenza n. 1146 del 1988)" (Bartole, 2015: 942; Tega, 2015: 1141; Panzeri, 2017: 727).

\section{LA CORTE COSTITUZIONALE INNANZI AI LIMITI DELLA DEMOCRAZIA RAPPRESENTATIVA}

La dimensione "politica" della giurisprudenza costituzionale ha trovato un terreno fertile allorché le sue decisioni hanno avuto a oggetto profili delicati della democrazia rappresentativa, come la richiesta da parte del corpo elettorale di una partecipazione diretta ai processi decisionali e la valutazione degli effetti distorsivi che le più recenti leggi elettorali producevano sul corretto rapporto tra esercizio del diritto di voto e formazione della rappresentanza politica. Nel primo caso, ha affrontato il tema della possibile convivenza tra assemblee parlamentari e istituti di democrazia deliberativa; nel secondo della compatibilità della struttura dei sistemi elettorali con i modi di formazione della democrazia rappresentativa ricavabili dalla Costituzione. Si tratta di problematiche che mettono in crisi il circuito della democrazia rappresentativa individuato dai nostri Costituenti, il quale faceva perno sul binomio suffragio

60 Così in sentenza n. 365 del 2007.

61 Sentenza n. 118 del 2015. 
universale e ruolo dei partiti politici come strumento per assicurare la partecipazione dei cittadini alla determinazione della politica nazionale ${ }^{62}$.

La Corte costituzionale —in sede di controllo di ammissibilità delle richieste di referendum abrogativo- ha individuato nel referendum un istituto spurio rispetto a un sistema imperniato sulla forma di governo parlamentare e sul ruolo centrale dei partiti politici: di conseguenza gli istituti di partecipazione diretta alle decisioni politiche sono stati valorizzati solo nel caso in cui il loro esercizio non fosse alternativo, ma complementare alla democrazia rappresentativa. Siffatto orientamento giurisprudenziale, d'altra parte, trovava un conforto sia nelle posizioni espresse nel corso dei lavori in Assemblea costituente, sia nelle soluzioni adottate dal Parlamento in sede di disciplina normativa degli istituti referendari.

Con riferimento al dibattito svoltosi durante i lavori dell'Assemblea costituente, un'atmosfera inizialmente favorevole ad ammettere varie forme di democrazia diretta perse progressivamente di consistenza a causa del manifestarsi di forti preoccupazioni per le conseguenze che un'applicazione distorta di tali istituti avrebbe potuto riverberare sulla funzionalità del sistema rappresentativo: tanto che il Presidente dell'Assemblea costituente, riassumendo gli esiti del dibattito, si era premurato di ricordare che anche i sostenitori più convinti del referendum riconoscevano l'opportunità di "cautele e regolazioni per fare un buono ed avveduto uso dell'istituto" (Luciani, 2005: 31; Nania, 1974: 662; Rolla, 2016: 207).

A sua volta, il legislatore - dopo avere tenuto per 22 anni questo istituto in una sorta di "limbo" a causa della mancata approvazione della legge di attuazione - ha introdotto con la legge n. 352 del 1970 stringenti limiti al fine di escludere che l'esercizio del referendum possa interferire con la democrazia rappresentativa. Basti considerare che gli artt. 31 e 34 di tale legge impediscono una sovrapposizione tra lo svolgimento del referendum e il rinnovo delle assemblee parlamentari; mentre il successivo art. 39, introducendo una clausola di improcedibilità della richiesta di referendum ${ }^{63}$, conferma la volontà del legislatore di individuare nel Parlamento il cardine del sistema di produzione delle norme di rango legislativo.

62 A questo proposito studi politologici hanno qualificato la grave crisi di rappresentatività delle istituzioni politiche ricorrendo all'espressione di "democrazie senza democrazia" (Salvadori, 2009: 10).

63 In base alla quale, se prima della data dello svolgimento del referendum, la legge o le singole disposizioni cui il referendum si riferisce sono state abrogate, l'Ufficio centrale per il referendum dichiara che le operazioni relative non hanno più corso. 
A sua volta la giurisprudenza costituzionale, proseguendo gli indirizzi sopra richiamati, ha svolto una funzione "creativa" finalizzata a enucleare dalla ratio dell' istituto limiti ulteriori rispetto a quelli inizialmente ipotizzati. In tal modo ha favorito una trasfigurazione del referendum abrogativo, limitandone selettivamente i modi di esercizio: la ratio unitaria che emerge dalla sua ampia giurisprudenza in materia è identificabile nella volontà di evitare che il referendum possa trasformarsi in uno strumento privilegiato di contrapposizione politica del corpo elettorale nei confronti della rappresentanza politica presente in Parlamento.

Nella sua azione di limitazione delle materie suscettibili di referendum abrogativo il giudice costituzionale non solo ha interpretato estensivamente le materie contenute nell'art. 75 Cost. ${ }^{64}$, ma ha anche individuato ulteriori limiti "impliciti", connessi al contenuto e alla struttura del quesito ${ }^{65}$.

Innanzitutto, sono state ritenute inammissibili le richieste di referendum il cui quesito è eterogeneo e privo di una matrice razionalmente unitaria, in quanto contraddicono la natura del referendum che si basa su di una pronuncia sintetica del corpo elettorale (sì o no) attorno ad un quesito specifico ${ }^{66}$. Quindi, la Corte ha individuato un'ulteriore causa di inammissibilità nei casi in cui il quesito, consistendo in una mirata selezione di singole parole, parti o commi della legge, ha la capacità di trasformare il referendum da abrogativo a propositivo ${ }^{67}$.

Infine, si è esclusa l'ammissibilità di referendum volti ad abrogare leggi riconducibili a due distinte tipologie: le leggi a contenuto costituzionalmente vincolato e quelle costituzionalmente necessarie. Anche se questi due tipi ten-

64 In tema di leggi tributarie il giudice costituzionale ha precisato che appartengono a tale categorie tutte le norme che determinano un prelievo forzoso da parte dello Stato delle risorse economiche dei contribuenti, finalizzato alla soddisfazione di pubblici bisogni; mentre nel tipo "leggi di bilancio" ha fatto rientrare tutte le norme che incidono sul quadro delle coerenze macroeconomiche e che sono funzionali a conseguire un equilibrio di bilancio. In tema di leggi di autorizzazione a ratificare trattati internazionali, la Corte ha ritenuto che dovessero rientrare nel divieto anche le leggi di esecuzione dei trattati internazionali regolarmente ratificati e quelle necessarie per dare attuazione agli obblighi internazionali.

Le linee-guida per valutare l'ammissibilità delle richieste di referendum abrogativo sono state fissate nella fondamentale sentenza n. 16 del 1978 e via via affinate nella successiva giurisprudenza Per ulteriori riferimenti si rinvia (Rolla, 2018)

66 Si veda, ad esempio, sentenza n. 49 del 2005. A proposito dei caratteri che, secondo la giurisprudenza costituzionale, deve possedere il quesito abrogativo: Carnevale, 1992: 159; Mangia, 1999: 281; Vizioli, 1998: 413; Barcellona, 2017.

67 Si vedano, ad esempio, le sentenze n. 47 del 1991 e n. n. 92 del 2012. 
dono talvolta a confondersi, si possono considerare a contenuto costituzionalmente vincolato le norme primarie la cui perdita di efficacia determinerebbe un'indiretta lesione di specifiche disposizioni costituzionali (come nel caso delle disposizioni che derivano da obblighi connessi all'adesione dell'Italia all'Unione europea); mentre appartengono, invece, alla categoria delle leggi costituzionalmente necessarie le norme primarie la cui abrogazione priverebbe totalmente di efficacia un principio o impedirebbe il funzionamento di un organo la cui esistenza è garantita dalla Costituzione ${ }^{68}$.

Tra le leggi costituzionalmente necessarie occupa una posizione particolare la legislazione in materia elettorale: in proposito, il giudice costituzionale ha sintetizzato le regole cui si debbono attenere i referendum in materia, sottolineando come le proposte di referendum non possono riguardare una legge elettorale nella sua interezza, e come i quesiti debbano essere formulati in modo che dall'esito del referendum residui, comunque, una normativa immediatamente applicabile, in modo da garantire la "costante operatività dell'organo" 69 .

Di recente, il giudice costituzionale ha dovuto misurarsi anche con la delicata problematica della legislazione elettorale —elemento portante della democrazia rappresentativa-: la sua giurisprudenza si è sviluppata lungo un sentiero normativo assai delicato in cui la linea di demarcazione tra discrezionalità politica e illegittimità non è sempre netta, così come i parametri costituzionali da utilizzare non sempre risultano univoci.

A partire dal 2005 il sistema politico è apparso difficilmente governabile, l'instabilità degli esecutivi si è accentuata e si è accresciuta la difficoltà di esprimere delle solide maggioranze: di conseguenza, il Parlamento ha tentato di affrontare il problema approvando nuove leggi elettorali che, per un verso, miravano, attraverso premi di maggioranza, a favorire la formazione di un indirizzo politico omogeneo e, per un altro verso, limitavano in misura significativa il potere dell'elettore di scegliere i propri rappresentanti. Tuttavia, queste leggi sono state dichiarate in più parti costituzionalmente illegittime, valorizzando il ruolo della Corte quale organo di mediazione nei conflitti "politici" (Minincleri, 2017; Ferri, 2017; Tondi, 2017; Baldini, 2017; Tarli, 2018).

68 Da ciò, ad esempio, il divieto di abrogazione totale di una legge necessaria per assicurare la protezione di diritti costituzionalmente garantiti; ovvero di leggi che regolano profili necessari inerenti alla composizione di organi costituzionali o di rilevanza costituzionale.

69 Si veda la sentenza n. 13 del 2012. Circa i problemi di ammissibilità dei referendum in materia elettorale: Lanchester, 1992; Biancolatte, 2008; Bin, 1999; Rolla, 2018. 
Nel 2014, il giudice costituzionale ha dichiarato costituzionalmente illegittime parti della legge n. 270 del 2005. In primo luogo, ha ritenuto che il premio di maggioranza previsto per l'elezione della Camera dei deputati e del Senato - suscettibile di trasformare una maggioranza anche esigua di voti in una maggioranza assoluta di seggi- non rispettasse i criteri di proporzionalità e di ragionevolezza, finendo per snaturare il principio fondamentale di eguaglianza del voto. Quindi, ha considerato illegittima la scelta del legislatore di rimettere interamente ai partiti la formazione delle liste e l'ordine delle candidature, in quanto non consentiva all'elettore di esprimere almeno una preferenza sui candidati da eleggere ${ }^{70}$.

Successivamente, nel 2017, la Corte ha dichiarato incostituzionale nei suoi elementi essenziali la legge elettorale n. 52 del 2015 nelle parti che disciplinavano il ballottaggio e la possibilità per i capilista di presentarsi in più collegi. La prima normativa (nella misura in cui potenzialmente consentiva di conseguire un premio di maggioranza anche a una lista che aveva avuto nel primo turno un consenso esiguo) ledeva il principio di eguaglianza sotto il profilo della ragionevolezza: infatti, la lista più votata aveva una sovra rappresentazione dei parlamentari rispetto ai voti effettivamente conseguiti. Invece, la possibilità per i capilista di presentarsi in numerosi collegi produceva, secondo il giudice costituzionale, un'alterazione del principio di rappresentanza in quanto riservava alla piena discrezionalità del capolista (che poteva liberamente decidere per quale collegio optare e "scegliere" i primi tra i non eletti che divenivano, di conseguenza, deputati) il destino del voto di preferenza espresso dall'elettore.

Nel motivare la propria decisione il giudice costituzionale ha utilizzato come parametro fondamentale il principio di ragionevolezza e di proporzionalità, ritenendo che se l'obiettivo perseguito dal legislatore era costituzionalmente corretto - favorire la formazione di maggioranze parlamentari omogenee-, invece le soluzioni individuate risultavano eccessivamente sbilanciate a danno di altri interessi costituzionali. Se con questa ratio decidendi la Corte entra nel merito delle soluzioni introdotte dal Parlamento, con un obiter dictum finale si fa carico delle possibili conseguenze di una dichiarazione di incostituzionalità, ribadendo il principio della continuità degli organi costituzionali. Pertanto il giudice costituzionale, precisando che gli effetti delle dichiarazioni d'incostituzionalità si produrranno "esclusivamente in occasione di una nuova consultazione elettorale", fa salvi tutti gli atti che le Camere hanno adottato prima dello svolgimento di nuove consultazioni elettorali: anche quelli di maggior rilevanza politica e costituzionale come una legge di riforma

70 Così in sentenza n. 1 del 2014. 
della Costituzione, l'elezione di giudici costituzionali e membri del Consiglio superiore della magistratura, l'approvazione della fiducia al governo, l'elezione del Presidente della Repubblica (Rescigno, 2014: 34; Giupponi et al., 2014: 629; D’Andrea, 2014: 44; Carnevale, 2013; Serges, 2014).

\section{Bibliografia}

Aja, E. (1998). Las tensiones entre el Tribunal Constitucional y el legislador en la Europa actual. Barcelona: Ariel.

Ambrosini, G. (1953). La Corte Costituzionale. Palermo. Aragón, M. (1986). ¿Estado jurisdiccional autonómico? Revista vasca de Administración Pública, 16, 7-22.

Barak, A. (2012). Proportionality. Constitutional Rights and their limitations. Cambridge: Cambridge University Press. Disponible en: https://doi.org/10.1093/ oxfordhb/9780199578610.013.0036.

Bartole, S. (1985). Le Regioni, le Province, i Comuni. Bologna: Zanichelli.

Baschieri, G., Bianchi d'Espinosa, L. y Gianattasio, C. (1949). La Costituzione italiana. Commento analitico. Firenze: Noccioli.

Battaglini, M. (1957). Contributi alla storia del controllo di costituzionalità delle leggi. Milano: Giuffrè.

Bernal Pulido, C. (2003). El principio de proporcionalidad y los derechos fundamentales. Madrid: Centro de Estudios Políticos y Constitucionales.

Bianchi, P. (2006). La garanzia dei diritti sociali nel dialogo tra legislatore e Corte costituzionale. Pisa: Plus.

Bin, R. (1992). Diritti e argomenti: il bilanciamento degli interessi nella giurisprudenza costituzionale. Milano: Giuffrè,

Biondi dal Monte, F. (2013). Dai diritti sociali alla cittadinanza. Torino: Giappichelli.

Bonini, F. (1996). Storia della Corte costituzionale. Firenze: La Nuova Italia.

Brancati, B. (2018). Tra diritti sociali e crisi economica. Un difficile equilibrio per le Corti costituzionali. Pisa: Pisa University Press.

Calamandrei, P. (1950). Lillegittimità costituzionale delle legge nel processo civile. Padova: CEDAM.

Calamandrei, P. y Levi, A. (1950). Commentario sistematico alla Costituzione italiana. Firenze: Barbera.

Cappelletti, M. (1955). La giurisdizione costituzionale delle libertà. Milano: Giuffrè.

Caretti, P. y Gardino, A. (1988). Giudici e Corte costituzionale nel sindacato sulle leggi. Milano: Giuffrè.

Carnevale, P. (1992). Il referendum abrogativo e i limiti alla sua ammissibilità nella giurisprudenza costituzionale. Padova: CEDAM.

Casonato, C. y Frosini, T. E. (2006). La fecondazione assistita nel diritto comparato. Torino: Giappichelli.

Ceccherini, E. (2008). La tutela della dignità delll'uomo. Napoli: Editoriale Scientifica. 
Crisafulli, V. (1993). Lezioni di diritto costituzionale, II. Padova: CEDAM.

Colapietro, C. (1990). Le sentenze additive e sostitutive della Corte costituzionale. Pisa: Pacini.

D’Andrea, L. (2005). Ragionevolezza e legittimazione del sistema. Milano: Giuffrè.

D’Atena, A. (1991). Costituzione e Regioni. Milano: Giuffrè.

De Siervo, U. (2008). L'istituzione della Corte costituzionale in Italia: dall'Assemblea costituente ai primi anni di attività della Corte, in La giustizia costituzionale tra memoria e prospettive. A cinquant'anni dalla pubblicazione della prima sentenza della Corte costituzionale. Torino: Giappichelli.

- (2015). La Corte costituzionale e il potere giudiziario. Torino: Giappichelli.

D’Orazio, G. (1981). La genesi della Corte costituzionale. Milano: Edizioni di Comunità.

Esposito, C. (1954). Autonomie locali e decentramento amministrativo nell'art.5 della Costituzione. Padova: CEDAM.

Fossas, E. y Requejo, F. (1999). Asimetría federal y estado plurinacional. El debate sobre la acomodación de la diversidad en Canadá, Bélgica y España. Madrid: Trotta.

García de Enterría, E. (1985). La Constitución como norma y el Tribunal constitucional. Madrid: Civitas.

Ghisalberti, M. (2000). Storia costituzionale d'Italia. Bari: Laterza.

Groppi, T. (2019). Interventi di terzi e amici curiae: dalla prospettiva comparata a uno sguardo sulla giustizia costituzionale in Italia. Disponible en: https://bit. ly/2X4cf1I.

Heintzen, M. (2012). Il principio di proporzionalità. Modena: Mucchi.

Luciani, M. (1984). Le decisioni processuali e la logica del giudizio costituzionale incidentale. Padova: CEDAM.

Mangia, A. (1999). Referendum. Padova: CEDAM.

Mannella, F. (2011). Giudici comuni e applicazione della Costituzione. Napoli: Editoriale Scientifica.

Masala, P. (2016). La tutela dei diritti sociali nelle situazioni di crisi economica: gli orientamenti della Corte costituzionale italiana. En Stato di diritto e crisi delle finanze pubbliche. Napoli: ESI.

Modugno, F. (1985). La Corte costituzionale italiana oggi, in Scritti sulla Giustizia costituzionale in onore di Vezio Crisafulli. Padova: CEDAM.

- (2007). La ragionevolezza nella giustizia costituzionale. Napoli: Editoriale Scientifica.

Mortati, C. (1972). Competenza esclusiva della Corte costituzionale a dichiarare linvalidità delle leggi anteriori alla Costituzione. Milano: Giuffrè.

Morrone, A. (2000). Il custode della ragionevolezza. Milano: Giuffrè.

- (2014). Il bilanciamento nello Stato costituzionale. Teoria e prassi nelle tecniche. Torino: Giappichelli.

Moscarini, A. (1996). Ratio legis e valutazioni di ragionevolezza della legge. Torino: Giappichelli.

Paladin, L. (1985). Diritto regionale. Padova: CEDAM. 
Palermo, F. y Woelk., J. (2008). Diritto costituzionale comparato dei gruppi e delle minoranze. Padova: CEDAM.

Passaglia, P. (2011). Le Juge constitutionnel et le Législateur. L'expérience italienne. Saarbruken: Editions Universitaires européennes.

Pasquino, P. y Randazzo, B. (2009). Come decidono le corti costituzionali (e le altre corti). Milano: Giuffrè.

Pegoraro, L. (1987). La Corte e il Parlamento: sentenze-indirizzo e attività legislativa. Padova: CEDAM.Pegoraro, P. (1998). Lineamenti di giustizia costituzionale comparata. Torino: Giappichelli.

Pegoraro, P. (1998). Lineamenti di giustizia costituzionale comparata. Torino: Giappichelli.

Pinardi, R. (2007). L’horror vacui nel giudizio sulle leggi. Milano: Giuffrè.

Pinelli, C. (2000). Prospettive di accesso alla giustizia costituzionale e nozione di giudice a quo. Torino: Giappichelli.

Pizzorusso, A. (1967). Le minoranze nel diritto pubblico interno. Milano: Giuffrè.

Pizzorusso, A. (1981). Art. 134. Commentario della Costituzione. Bologna: Zanichelli. Poggeschi, G. (2010). Diritti linguistici. Un'analisi comparata. Roma: Carocci.

Politi, F. (1997). Gli effetti nel tempo delle sentenze di accoglimento della Corte costituzionale: contributo ad una teoria dell'invalidità costituzionale delle leggi. Padova: CEDAM.

Rolla, G. (1982). La determinazione delle materie di competenza regionale nella giurisprudenza della Corte costituzionale. Le Regioni, 100-123.

- (1995). Il processo di ibridazione dei sistemi accentrati di giustizia costituzionale. Note di diritto comparato, in Estado constitucional, derechos humanos, justicia y vida universitaria. México: UNAM.

- (2002). Relazioni tra ordinamenti e sistema delle fonti .Considerazioni alla luce della legge costituzionale 3/01. Le Regioni, 30 (2-3), 321-350.

- (2007). Scritti sulla giustizia costituzionale. Genoa: ECIG.

- (2008). L'autonomia delle comunità territoriali. Profili costituzionali. Milano: Giuffrè.

- (2010). L'interpretazione adeguatrice tra Tribunale costituzionale e giudici comuni in Spagna. Estudios constitucionales, 8 (2), 601-632. Disponible en: https://doi.org/10.4067/S0718-52002010000200018.

- (2012). La tutela dei diritti fondamentali. Roma: Carocci.

- (2012). L'influenza delle Carte sovranazionali sulla configurazione legale dei diritti e i lineamenti del sistema di giustizia costituzionale. Politica del Diritto, $43(2-3), 181-205$.

- (2017). L'evoluzione dei sistemi accentrati di giustizia costituzionale. Note di diritto comparato. En S. Bagni, G. A. Figueroa Mejía, G. Pavani (coords.). La ciencia del derecho constitucional comparado, II (pp. 839-910). México: Tirant lo Blanc.

- (2018). L'organizzazione costituzionale dello Stato. Milano: Giuffrè.

- (2019). Federalismo e Regionalismo in Europa. Alcune riflessioni sulle dinamiche in atto. Diritto Pubblico Comparado ed Europeo, 21, 685-710. 
- (2019). L'evoluzione dello Stato regionale in Italia: tra crisi del regionalismo omogeneo e aspirazioni a un'autonomia asimmetrica dei territori. Le Regioni, 1, 73-97.

Romboli, R. (2017). Ricordando Alessandro Pizzorusso. Il pendolo della Corte. Le oscillazioni della Corte costituzionale tra l'anima 'politica' e quella 'giurisdizionale'. Torino: Giappichelli.

Ruggeri, A. (1988). Le attività “conseguenziali” nei rapporti fra la Corte costituzionale e il legislatore. Milano: Giuffrè.

Salazar, C. (2000). Dal riconoscimento alla garanzia dei diritti sociali: orientamenti e tecniche decisorie della Corte costituzionale a confronto. Torino: Giappichelli.

- (2015). Crisi economica e diritti fondamentali, in Spazio costituzionale e crisi economica. Napoli: Jovene.

Salerno, M. G. (1992). Il Referendum. Padova: CEDAM.

Scaccia, G. (2000). Gli "strumenti" della ragionevolezza nel giudizio costituzionale. Milano: Giuffrè.

Salvadori, M. L. (2009). Democrazie senza democrazia. Bari: La Terza.

Tarli Barbieri, G. (2018). La legislazione elettorale nell'ordinamento italiano. Milano: Giuffrè.

Toniatti, R. (2003). Il regionalismo relazionale e il governo delle reti: primi spunti ricostruttivi. En Il nuovo ordinamento regionale. Milano: Giuffrè.

Veronesi, P. (2007). Il corpo e la Costituzione. Milano: Giuffrè. 